\title{
Emission from $\mathrm{HCN}$ and $\mathrm{CH}_{3} \mathrm{OH}$ in comets
}

\section{Onsala 20-m observations and radiative transfer modelling}

\author{
P. Bergman ${ }^{1}$, M. S. Lerner ${ }^{1}$, A. O. H. Olofsson ${ }^{1}$, E. Wirström ${ }^{1}$, J. H. Black ${ }^{1}$, P. Bjerkeli ${ }^{1}$, R. Parra ${ }^{2}$, and K. Torstensson ${ }^{2}$ \\ ${ }^{1}$ Department of Space, Earth and Environment, Chalmers University of Technology, Onsala Space Observatory, 43992 Onsala, \\ Sweden \\ e-mail: per.bergman@chalmers.se \\ ${ }^{2}$ European Southern Observatory, Av. Alonso de Cordova 3107, Vitacura, Santiago, Chile
}

Received 4 November 2021 / Accepted 1 February 2022

\begin{abstract}
Aims. The aim of this work is to characterise $\mathrm{HCN}$ and $\mathrm{CH}_{3} \mathrm{OH}$ emission from recent comets.

Methods. We used the Onsala 20-m telescope to search for millimetre transitions of HCN towards a sample of 11 recent and mostly bright comets in the period from December 2016 to November 2019. Also, $\mathrm{CH}_{3} \mathrm{OH}$ was searched for in two comets. The HCN sample includes the interstellar comet 2I/Borisov. For the short-period comet 46P/Wirtanen, we were able to monitor the variation of HCN emission over a time-span of about one month. We performed radiative transfer modelling for the observed molecular emission by also including time-dependent effects due to the outgassing of molecules.

Results. HCN was detected in six comets. Two of these are short-period comets and four are long-period. Six methanol transitions were detected in 46P/Wirtanen, enabling us to determine the gas kinetic temperature. From the observations, we determined the molecular production rates using time-dependent radiative transfer modelling. For five comets, we were able to determine that the $\mathrm{HCN}$ mixing ratios lie near $0.1 \%$ using contemporary water production rates, $Q_{\mathrm{H}_{2} \mathrm{O}}$, taken from other studies. This $\mathrm{HCN}$ mixing ratio was also found to be typical in our monitoring observations of 46P/Wirtanen but here we notice deviations of up to $0.2 \%$ on a daily timescale which could indicate short-time changes in outgassing activity. From our radiative transfer modelling of cometary comae, we find that time-dependent effects on the HCN level populations are of the order of 5-15\% when $Q_{\mathrm{H}_{2} \mathrm{O}}$ is around $2 \times 10^{28} \mathrm{~mol} \mathrm{~s}{ }^{-1}$. The effects may be stronger for comets with lower $Q_{\mathrm{H}_{2} \mathrm{O}}$. The exact details of the time-dependent effects depend on the amount of neutral and electron collisions, radiative pumping, and molecular parameters such as the spontaneous rate coefficient.
\end{abstract}

Key words. comets: general - radio lines: general

\section{Introduction}

Most comets are expected to be remnants from the early times, some 4.5 billion years ago, when the Solar System was formed, and therefore may contain records of the chemical and physical properties of these times past. Exceptions to this notion are the recent discoveries of the objects 1I/'Oumuamua and 2I/Borisov which are suggested to be interstellar visitors entering our Solar System with strongly hyperbolical and thus unbound trajectories. When approaching the Sun to within a few astronomical units (au), comets can start to sublimate molecules and other volatile particles from their surfaces and form what is known as a cometary coma. Studying the composition of the content of cometary comae can therefore reveal information about the physical conditions and chemistry prevailing when the Solar System was born or that of similar systems in the solar neighbourhood in the case of interstellar visitors.

Studies of the molecular content of cometary comae are performed either by remote sensing (mainly by recording spectral signatures over a wide range of wavelengths from radio to the ultraviolet regimes) or by in situ measurements made by spacecraft like Giotto (1P/Halley) and, more recently, Rosetta (67P/Churyumov-Gerasimenko). As pointed out by Rubin et al. (2019), most abundances of the volatile content, as determined by these various means over the last $40 \mathrm{yr}$, are reminiscent of those in the interstellar medium (ISM) and thus suggest a chemical origin in interstellar, star-forming material. The major gaseous constituent of the neutral inner coma is water molecules, and the abundances of other species relate to water via the mixing ratio. For instance, the next two most abundant species, $\mathrm{CO}_{2}$ and CO, constitute typically $10-20 \%$ of that of water (Mumma \& Charnley 2011; Rubin et al. 2019). Further out in the coma, the solar radiation will affect the composition. At 1 au from the Sun, the outgassing water molecules are photodissociated into $\mathrm{H}$ and $\mathrm{OH}$ at a rate of about $(1-2) \times 10^{-5} \mathrm{~s}^{-1}$ (Huebner et al. 1992) where the factor of two variation reflects the degree of solar activity. Another water-destruction mechanism is photoionization into $\mathrm{H}_{2} \mathrm{O}^{+}$, which is about 30 times slower. This latter process, together with photoionization of $\mathrm{OH}$, previously formed from water, is the main source of electrons in the coma (Rubin et al. 2009). As water is relatively difficult to spectroscopically observe, several other, indirect methods have been employed to determine the water production rate. Firstly, observations of the photodissociation product $\mathrm{OH}$ can be used as a proxy for water (Despois et al. 1981; Bonev et al. 2006). Secondly, observations of the remaining $\mathrm{H}$, via Ly- $\alpha$, can also be used as exemplified by the Solar Wind ANisotropies (SWAN) Ly- $\alpha$ camera observations on board the SOlar and Heliospheric Observer (SOHO) spacecraft (e.g. Combi et al. 2011). A third indirect method is to use a relatively abundant parent species, like $\mathrm{HCN}$, as an indicator of water; see Mumma \& Charnley (2011) for an overview. Direct observations of the ground-state 
Table 1. Observed lines.

\begin{tabular}{lccccc}
\hline \hline Molecule & $\begin{array}{c}\text { Frequency } \\
(\mathrm{MHz})\end{array}$ & Transition & $g_{\mathrm{u}}$ & $\begin{array}{c}E_{\mathrm{u}}{ }^{(b)} \\
(\mathrm{K})\end{array}$ & $\begin{array}{c}A_{\mathrm{ul}}(c) \\
\left(\mathrm{s}^{-1}\right)\end{array}$ \\
\hline $\mathrm{HCN}$ & 88630.42 & $J_{F}=1_{1}-0_{1}$ & 3 & 4.2 & $2.4 \times 10^{-5}$ \\
& 88631.85 & $J_{F}=1_{2}-0_{1}$ & 5 & 4.2 & $2.4 \times 10^{-5}$ \\
& 88633.94 & $J_{F}=1_{0}-0_{1}$ & 1 & 4.2 & $2.4 \times 10^{-5}$ \\
\hline $\mathrm{CH}_{3} \mathrm{OH}$ & 95169.39 & $J_{K}=8_{0}-7_{1} A^{+}$ & 17 & 83.5 & $4.3 \times 10^{-6}$ \\
& 95914.31 & $J_{K}=2_{1}-1_{1} A^{+}$ & 5 & 21.4 & $2.5 \times 10^{-6}$ \\
& 96739.36 & $J_{K}=2_{-1}-1_{-1} E$ & 5 & 12.5 & $2.6 \times 10^{-6}$ \\
& 96741.37 & $J_{K}=2_{0}-1_{0} A^{+}$ & 5 & 7.0 & $3.4 \times 10^{-6}$ \\
& 96744.54 & $J_{K}=2_{0}-1_{0} E$ & 5 & 20.1 & $3.4 \times 10^{-6}$ \\
& 96755.50 & $J_{K}=2_{+1}-1_{+1} E$ & 5 & 28.0 & $2.6 \times 10^{-6}$ \\
\hline
\end{tabular}

Notes. ${ }^{(a)}$ Frequencies are taken from Pickett et al. (1998). ${ }^{(b)}$ Upper energy for methanol $E$-species includes the $7.9 \mathrm{~K}$ offset relative to the $J_{K}=0_{0}$ level of the $A$-species. ${ }^{\left({ }^{c}\right)} A$-coefficients from Müller et al. (2001).

water lines are scarce and are mostly limited to satellites (e.g. Lecacheux et al. 2003; Lis et al. 2013; Biver et al. 2015). Infrared spectroscopy of the ro-vibrational water lines has proven useful in determining the water production rates (Mumma et al. 2003). This latter method, albeit relying on fluorescence pumping models, has the advantage that the excitation conditions can be determined via a rotation temperature, and consequently provide more accurate production rates (e.g. DiSanti et al. 2016). Lastly, in situ measurements by spacecraft via high-resolution mass spectroscopy provide perhaps the most direct way to probe the molecular coma content, although this method is limited to only a few comets (e.g. Läuter et al. 2020).

The observations presented in this paper are part of an ongoing effort to study comets at millimetre $(\mathrm{mm})$ wavelengths using the Onsala Space Observatory (OSO) 20-meter telescope. This effort was initiated by Wirström et al. (2016) who presented HCN(1-0) observations of the long-period comets C/2013 R1 (Lovejoy) and C/2014 Q2 (Lovejoy). We expand this study here with observations of another 12 comets. The observational efforts focus primarily on using $\mathrm{HCN}(1-0)$ as an indicator of water, but one comet, 46P/Wirtanen, was also observed in methanol. Both of these molecules are believed to be released from nucleus ices (Dello Russo et al. 2016). Our comet target sample comprises bright comets with perihelion dates from late 2016 to 2019. The sample includes comets belonging to the Jupiter family as well as the Oort cloud. In addition, we searched for HCN towards 2I/Borisov. Furthermore, we also investigate possible caveats - in terms of time-dependent radiative transfer effects - that may complicate the interpretation of the observed emission from $\mathrm{HCN}$ (as well as from other molecules) in cometary comae.

This paper is structured as follows. In the following section, we describe the observations made with the OSO 20-m telescope. In Sect. 3 we present the results and in Sect. 4 we outline the radiative transfer analysis made in which we also take into account time-dependent aspects. After discussing our results in Sect. 5, we finally present our conclusions.

\section{Observations}

All observations presented in this paper were made from late 2016 until late 2019 using the radome-enclosed OSO 20-m antenna equipped with the $3 \mathrm{~mm}$ channel of the 3 and $4 \mathrm{~mm}$ receiver system (Belitsky et al. 2015). The radome allows us to observe sources near the Sun without thermal distortion of the telescope optics. The rest frequencies of the target lines (see Table 1) were covered by two frequency tunings, one centred near $88 \mathrm{GHz}$ and the other near $96 \mathrm{GHz}$. All tunings were performed in the velocity frame of the comet. Coordinates and velocities of all comets were taken from the Horizons system ${ }^{1}$ (Giorgini et al. 1996) tabulated every hour and then interpolated by the observing system. The antenna half-power beam width is 40 arcsec at the HCN frequency and about 37 arcsec for the $\mathrm{CH}_{3} \mathrm{OH}$ observations.

The Fast Fourier Transform Spectrometers (FFTSs) were configured into a wide $4-\mathrm{GHz}$ mode or a narrow $156 \mathrm{MHz}$ mode. In both modes, the two linear polarizations are recorded separately. The wide 4-GHz mode is made up of two partly overlapping $2.5 \mathrm{GHz}$ FFTSs of 32768 channels and was used for the $\mathrm{CH}_{3} \mathrm{OH}$ observations and part of the $\mathrm{HCN}$ observations. The wide mode results in a velocity resolution of about $0.25 \mathrm{~km} \mathrm{~s}^{-1}$. The narrow $156 \mathrm{MHz}$ setup was routinely used for the HCN observations. The observations were made in frequency switching mode with a frequency throw for the $\mathrm{HCN}$ setup of $5 \mathrm{MHz}$ for data taken in 2016-2017 and 7 MHz from 2018 onwards. When observing $\mathrm{CH}_{3} \mathrm{OH}$, the wide spectrometer setup was employed to cover all lines (see Table 1) and the used frequency throw was larger, $25 \mathrm{MHz}$, to avoid line confusion.

The weather conditions varied during our observations. In the best conditions, the system temperature was around $120 \mathrm{~K}$. Typically the measurements were automatically halted when the system temperature became large, around 800-1200 K. Pointing and focus optimisations were performed several times a day using the $\mathrm{SiO} 2-1 v=1$ maser line at $86 \mathrm{GHz}$ towards stars. Measurements from 2018 onwards were made with the new antenna control system developed by M. Lerner and called BIFROST $^{2}$, which provides an improved and more automated control of remote observations. We used these capabilities to perform pointings and to refocus automatically every three hours

\footnotetext{
1 HORIZONS is a service of the Solar System Dynamics group at the NASA/Jet Propulsion Laboratory for computing ephemerides of solarsystem bodies. The web interface is at https://ssd.jpl.nasa.gov/ horizons.cgi

2 See https://www. chalmers.se/en/researchinfrastructure/ oso/radio-astronomy/20m/Pages/Handbook . aspx and http:// wwW.ira.inaf.it/eratec/florence/presentations/florens_ Lerner.pdf
} 
Table 2. Observed comets with the OSO 20-m telescope.

\begin{tabular}{lccccc}
\hline \hline Comet & Observing UT date range & $\begin{array}{c}\text { Int. time } \\
(\mathrm{h})\end{array}$ & $\begin{array}{c}R_{\mathrm{h}} \\
(\mathrm{au})\end{array}$ & $\begin{array}{c}\Delta \\
(\mathrm{au})\end{array}$ & $\begin{array}{c}d \\
(\mathrm{~km})\end{array}$ \\
\hline C/2016 U1 (NEOWISE) & $2016-12-22-2017-01-10$ & 14 & $0.71-0.34$ & $0.75-1.07$ & $5.2 \times 10^{4}$ \\
45P/Honda-Mrkos-Pajdušáková & $2017-01-18-2017-02-06$ & 31 & $0.65-0.90$ & $0.36-0.11$ & $7.0 \times 10^{3}$ \\
2P/Encke & $2017-03-04-2017-03-17$ & 15 & $0.37-0.38$ & $0.75-0.68$ & $4.1 \times 10^{4}$ \\
41P/Tuttle-Giacobini-Kresák & $2017-04-04-2017-04-24$ & 170 & $1.05-1.06$ & $0.14-0.17$ & $8.7 \times 10^{3}$ \\
C/2015 ER61 (PanSTARRS) & $2017-04-06-2017-05-09$ & 12 & $1.19-1.04$ & $1.21-1.23$ & $7.1 \times 10^{4}$ \\
C/2017 E4 (Lovejoy) & $2017-04-07$ & 8 & 0.63 & 0.67 & $3.9 \times 10^{3}$ \\
C/2015 V2 (Johnson) & $2017-05-18-2017-05-19$ & 13 & 1.67 & 0.87 & $5.0 \times 10^{4}$ \\
C/2017 O1 (ASASSN1) & $2017-10-12-2017-10-15$ & 30 & 1.50 & $0.73-0.72$ & $4.2 \times 10^{4}$ \\
96P/Machholz & $2017-10-30$ & 5 & 0.16 & 1.04 & $6.0 \times 10^{4}$ \\
46P/Wirtanen & $2018-12-08-2019-01-18$ & 232 & $1.06-1.16$ & $0.096-0.22$ & $4.7 \times 10^{3}$ \\
C/2018 Y1 (Iwamoto) & $2019-02-04-2019-02-19$ & 38 & $1.29-1.30$ & $0.46-0.39$ & $2.4 \times 10^{4}$ \\
2I/Borisov & $2019-10-28-2019-11-18$ & 89 & $2.21-2.06$ & $2.66-2.18$ & $1.4 \times 10^{5}$ \\
\hline
\end{tabular}

Notes. Towards 96P/Machholz only $\mathrm{CH}_{3} \mathrm{OH}$ was searched for. Both $\mathrm{HCN}$ and $\mathrm{CH}_{3} \mathrm{OH}$ were observed in $46 \mathrm{P} /$ Wirtanen. For the rest of the comets, only HCN was targeted.

and to automatically pause observations when the system temperature rose above $800 \mathrm{~K}$. We used 60 and $120 \mathrm{~s}$ integrations for individual scans. With the switch to BIFROST we employed observing blocks consisting of fifteen 60 -s scans with hot-load calibrations between every fifth scan.

Information on the 12 comets investigated in this study is summarized in Table 2 where the range of observing dates, total integration time, heliocentric distance, $R_{\mathrm{h}}$, distance to Earth, $\Delta$, and variations over the observing dates are also listed. The projected nucleocentric distance (near the centre of the date range), $d$, as subtended by the beam size for $\mathrm{HCN}(1-0)$ transition is also listed.

\section{Results}

The $\mathrm{HCN}$ and $\mathrm{CH}_{3} \mathrm{OH}$ observations are displayed in Figs. 1-7. The shown spectra have been smoothed to a resolution of $0.3 \mathrm{~km} \mathrm{~s}^{-1}$ (except the 46P/Wirtanen global average spectrum) and intensities are shown in $T_{\mathrm{mb}}$-scale. The individual scans were first folded before being noise-weighted to form an average. Because of the frequency-switching observing mode, a rather high-order polynomial was needed to subtract the baseline. The used baseline order was typically from 3 to 7 where the higher orders were used for the observations with larger frequency throws. The channel RMS and integrated line intensities, $I_{\mathrm{mb}}$, have been entered in Table 3. Errors and upper limits are $1 \sigma$ in this table. The variation of errors and limits among the comets is a combination of system temperature and integration time. The integrated line intensity of $\operatorname{HCN}(J=1 \rightarrow 0)$ is calculated as a sum of the three hyperfine structure (hfs) line intensities (listed in Table 1) using a box of $2 \mathrm{~km} \mathrm{~s}^{-1}$ in width centred on each hfs line. The 46P/Wirtanen monitoring measurements are listed in Table 4 as a function of UT date and modified Julian date (MJD). Below we give a short introduction and summary of the results for each comet observed:

- C/2016 U1 (NEOWISE). This comet (hereafter U1), discovered in October 2016 by the Near-Earth Object Wide-field Infrared Survey Explorer (NEOWISE), follows a slightly hyperbolic orbit (eccentricity just above 1). At perihelion on 14 January 2017 its heliocentric distance was 0.32 au. The spectrum presented in Fig. 1 is the average over four observing dates
Table 3. Observed HCN intensities and upper limits.

\begin{tabular}{lrc}
\hline \hline Comet & $\begin{array}{r}\text { Channel rms } \\
(\mathrm{mK})\end{array}$ & $\begin{array}{c}I_{\mathrm{mb}} \\
\left(\mathrm{mK} \mathrm{km} \mathrm{s}^{-1}\right)\end{array}$ \\
\hline C/2016 U1 (NEOWISE) & 7.5 & $<11.2$ \\
45P/Honda-Mrkos-Pajdušáková & 9.8 & $<14.7$ \\
2P/Encke & 12.9 & $<19.4$ \\
41P/Tuttle-Giacobini-Kresák & 3.8 & $23.8 \pm 5.1$ \\
C/2015 ER61 (PanSTARRS) & 10.0 & $49.3 \pm 13.4$ \\
C/2017 E4 (Lovejoy) & 17.3 & $<23.2$ \\
C/2015 V2 (Johnson) & 12.5 & $65.0 \pm 16.7$ \\
C/2017 O1 (ASASSN1) & 5.0 & $48.8 \pm 6.6$ \\
C/2018 Y1 (Iwamoto) & 5.5 & $68.2 \pm 7.3$ \\
2I/Borisov & 4.0 & $<5.5$ \\
\hline
\end{tabular}

ranging from 22 December 2016 to 10 January 2017. This object was not detected in $\mathrm{HCN}$ and the upper limit of the integrated intensity is listed in Table 3.

- 45P/Honda-Mrkos-Pajdušáková. This short-period comet discovered in 1948, and hereafter referred to as 45P, belongs to the Jupiter family and has a period of $5.26 \mathrm{yr}$. The water production was estimated by Dello Russo et al. (2020) to be $(2-3) \times$ $10^{27} \mathrm{~mol} \mathrm{~s}^{-1}$ using infrared spectroscopy in mid-February 2017. The same study reports an $\mathrm{HCN}$ production rate of $(3-4) \times$ $10^{24} \mathrm{~mol} \mathrm{~s}^{-1}$. On 16 February 2017, there was also a CN outburst seen (Springmann et al. 2019). We did not detect the HCN 1-0 triplet; see Fig. 1. The bulk of our HCN data was obtained early February 2017.

$-2 \mathrm{P} /$ Encke. This short-period comet was observed several times in the first half of March 2017. Our HCN observations resulted in a non-detection. Later in March, after perihelion, the water production rate was estimated to be $(3-4) \times 10^{28} \mathrm{~mol} \mathrm{~s}^{-1}$ and the HCN production rate was $(3-6) \times 10^{25} \mathrm{~mol} \mathrm{~s}^{-1}$ (Roth et al. 2018).

- 41P/Tuttle-Giacobini-Kresák. This comet was first observed in 1858 and later rediscovered in 1907 and 1952 as pointed out by Schleicher et al. (2019). Like 45P, this comet (hereafter 41P) belongs to the Jupiter family and has a period of 5.42 yr. In early April 2017 its peak water production rate 
Table 4. 46P/Wirtanen HCN 2018/19 monitoring results.

\begin{tabular}{cccccc}
\hline \hline Date & MJD & $\begin{array}{c}R_{\mathrm{h}} \\
(\mathrm{au})\end{array}$ & $\begin{array}{c}\Delta \\
(\mathrm{au})\end{array}$ & $\begin{array}{c}I_{\mathrm{mb}} \\
\mathrm{mK} \mathrm{km} \mathrm{s}^{-1}\end{array}$ & $\begin{array}{c}Q_{\mathrm{HCN}} / 10^{25} \\
\left(\mathrm{~mol} \mathrm{~s}^{-1}\right)\end{array}$ \\
\hline 1209 & 58461.8 & 1.056 & 0.088 & $135 \pm 19$ & $1.57 \pm 0.21$ \\
1210 & 58462.1 & 1.056 & 0.086 & $144 \pm 52$ & $1.65 \pm 0.60$ \\
1214 & 58466.7 & 1.056 & 0.078 & $135 \pm 11$ & $1.41 \pm 0.11$ \\
1215 & 58467.5 & 1.056 & 0.078 & $108 \pm 10$ & $1.10 \pm 0.10$ \\
1216 & 58468.5 & 1.056 & 0.077 & $78 \pm 13$ & $0.76 \pm 0.12$ \\
1217 & 58469.1 & 1.057 & 0.078 & $88 \pm 19$ & $0.88 \pm 0.18$ \\
1220 & 58472.7 & 1.060 & 0.081 & $145 \pm 11$ & $1.57 \pm 0.12$ \\
1221 & 58473.5 & 1.062 & 0.083 & $101 \pm 7$ & $1.08 \pm 0.07$ \\
1222 & 58474.2 & 1.063 & 0.085 & $84 \pm 10$ & $0.89 \pm 0.10$ \\
1224 & 58476.8 & 1.067 & 0.091 & $85 \pm 11$ & $0.96 \pm 0.12$ \\
1225 & 58477.5 & 1.069 & 0.095 & $96 \pm 7$ & $1.14 \pm 0.08$ \\
1226 & 58478.5 & 1.071 & 0.098 & $77 \pm 13$ & $0.91 \pm 0.15$ \\
1227 & 58479.2 & 1.074 & 0.102 & $110 \pm 17$ & $1.41 \pm 0.21$ \\
1228 & 58480.8 & 1.076 & 0.106 & $77 \pm 13$ & $0.97 \pm 0.16$ \\
1229 & 58481.5 & 1.079 & 0.111 & $115 \pm 21$ & $1.59 \pm 0.28$ \\
1230 & 58482.5 & 1.082 & 0.115 & $75 \pm 7$ & $1.01 \pm 0.09$ \\
1231 & 58483.5 & 1.085 & 0.120 & $104 \pm 12$ & $1.51 \pm 0.17$ \\
0101 & 58484.5 & 1.088 & 0.125 & $73 \pm 5$ & $1.05 \pm 0.07$ \\
0102 & 58485.2 & 1.091 & 0.130 & $65 \pm 6$ & $0.95 \pm 0.07$ \\
0110 & 58493.7 & 1.123 & 0.173 & $40 \pm 14$ & $0.67 \pm 0.23$ \\
0111 & 58494.2 & 1.128 & 0.179 & $44 \pm 11$ & $0.77 \pm 0.19$ \\
0117 & 58500.8 & 1.158 & 0.215 & $50 \pm 8$ & $1.02 \pm 0.16$ \\
0118 & 58501.2 & 1.164 & 0.222 & $28 \pm 6$ & $0.52 \pm 0.11$ \\
\hline & & & & & \\
\hline
\end{tabular}

Notes. Date is UT start of observation 2018/19mmdd. MJD corresponds to the centre of the time interval. See Sect. 4.2 for the HCN production rates.

was $3.5 \times 10^{27} \mathrm{~mol} \mathrm{~s}^{-1}$ (Moulane et al. 2018). The 41P nucleus rotation underwent an unusually large slow-down with a rotation period increasing from $20 \mathrm{~h}$ in March 2017 to more than $50 \mathrm{~h}$ in early May (Schleicher et al. 2019). Our April 2017 HCN 1-0 detection at the $4.6 \sigma$ level is shown in Fig. 1.

- C/2015 ER61 (PanSTARRS). This comet, hereafter ER61, follows a highly eccentric orbit and is suggested to originate in the inner Oort cloud (Meech et al. 2017). The OSO 20-m HCN $3.7 \sigma$ detection is shown in Fig. 2. The spectrum is an average over three observing days in 2017: 6, 11, and 22 April. The observations took place after ER61 underwent an outburst on 4 April (Opitom et al. 2019). Sekanina (2017) suggests that the flare-up was caused by fragmentation of the nucleus creating a companion nucleus which was observed later in June. Our observations on 9 May were disregarded because of an inaccurate set of ephemerides. Saki et al. (2021) report a water production rate of around $1 \times 10^{29} \mathrm{~mol} \mathrm{~s}^{-1}$ in mid-April 2017 and an HCN rotational temperature near $70 \mathrm{~K}$. This is consistent with the Atacama Large Millimeter/Submillimeter Array (ALMA) compact array HCN observations in mid-April by Roth et al. (2021b). Both studies determined the HCN production rate to be near $9 \times 10^{25} \mathrm{~mol} \mathrm{~s}^{-1}$.

- C/2017 E4 (Lovejoy). This long-period comet (referred to as E4) appears to have originated in the inner Oort Cloud (Faggi et al. 2018). Our observations took place on 7 April and resulted in an upper limit; see Fig. 2. Faggi et al. (2018) reported a water production rate of about $3 \times 10^{28} \mathrm{~mol} \mathrm{~s}^{-1}$ and an $\mathrm{HCN}$ production rate of about $5 \times 10^{25} \mathrm{~mol} \mathrm{~s}^{-1}$ only 3 days before our observations took place. The comet disintegrated in late April 2017.

- C/2015 V2 (Johnson). This Oort cloud comet (OCC), hereafter referred to as V2, follows a slightly hyperbolic orbit and was observed during one observing run stretching from 18-19 May in 2017 before the perihelion on 12 June. These observations resulted in an HCN $3.9 \sigma$ detection; see Fig. 2. Combi et al. (2021) estimated water production rates from SOHO/SWAN observations but to the best of our knowledge no other direct molecular studies of $\mathrm{V} 2$ are found in the literature for this period of time.

- C/2017 O1 (ASASSN1). This long-period comet, hereafter referred to as O1, is a possible Manx comet (Brinkman 2020), which is a category of tail-less long-period comets that may have formed in the inner Solar System before being ejected to the outskirts. Our observation, resulting in a $7.4 \sigma$ detection (Fig. 2), took place in two shifts in mid-October 2017 (12/13 and 14/15).

- 96P/Machholz. This short-period comet has a rather peculiar high-inclination, low-perihelion orbit, being a Jupiter-family comet (JFC). Its nucleus is thought to be inactive (e.g. Eisner et al. 2019). Our observations, of $\mathrm{CH}_{3} \mathrm{OH}$ only, were made when 96P/Machholz was at a heliocentric distance of 0.16 au on 30 October 2017 (the perihelion distance was 0.12 au for the 2017 apparition). Emission from $\mathrm{CH}_{3} \mathrm{OH}$ was not detected with the OSO 20-m at this date, see Fig. 3, and the upper limit for the $J_{K}=2_{0}-1_{0} A^{+}$line has been included in Table 5 .

$-46 \mathrm{P} /$ Wirtanen. This JFC was the only comet in our sample that could be monitored in HCN; see Fig. 5 and Table 4. The first detection of HCN was made on 9 December 2018 and the last detection was made on 18 January 2019. The detection level was better than $3 \sigma$ except on the dates 10 December and 10 January. On 20 December, we detected the strongest HCN line intensity. The HCN spectrum of this date is shown in Fig. 4. $\mathrm{CH}_{3} \mathrm{OH}$ was also clearly detected, and the spectrum (an average of data taken between 22 and 28 December) is shown in Fig. 6. Six different methanol lines around $96 \mathrm{GHz}$ are seen (Table 5), each at a detection level of $5 \sigma$ or better. 46P/Wirtanen came very close to Earth in December 2018 (0.08 au) and was therefore widely observed ${ }^{3}$. In addition, it showed much higher activity than during previous apparitions (Farnham et al. 2019). HCN was observed by Wang et al. (2020) $(J=1-0)$ and Coulson et al. (2020) $(J=4-3)$. The latter study also included $J=7-6$ $\mathrm{CH}_{3} \mathrm{OH}$ data. A week before its closest approach to Earth, Roth et al. (2021c) observed the $J=5-4 \mathrm{CH}_{3} \mathrm{OH}$ lines using the ALMA array. These authors found variable $\mathrm{CH}_{3} \mathrm{OH}$ outgassing consistent with the rotational period of $9 \mathrm{~h}$ (Farnham et al. 2021) of the nucleus. In December 2018, Biver et al. (2021) performed a molecular survey of 46P mainly using the Institut de RadioAstronomie Millimétrique (IRAM) 30-m telescope but also the NOrthern Extended Millimeter Array (NOEMA). At perihelion (12.9 December 2018), Moulane et al. (2019) reported a water production rate of $7.2 \times 10^{27} \mathrm{~mol} \mathrm{~s}^{-1}$ followed by a number of other infrared spectroscopy studies (Saki et al. 2020; Roth et al. 2021a; Bonev et al. 2021; Khan et al. 2021; McKay et al. 2021) as well as submillimetre observations of $\mathrm{H}_{2}^{18} \mathrm{O}$ (Lis et al. 2019).

- C/2018 Y1 (Iwamoto). This bright and long-period comet, hereafter called Y1, came close (0.4 au) to Earth in February 2018. On February 5, DiSanti et al. (2019) report $\mathrm{H}_{2} \mathrm{O}$ and $\mathrm{HCN}$ production rates of $2 \times 10^{28} \mathrm{~mol} \mathrm{~s}^{-1}$ and $4 \times 10^{25} \mathrm{~mol} \mathrm{~s}^{-1}$, respectively. By averaging data between 10 and 19 February we obtained a very clear $9 \sigma$ detection of $\mathrm{HCN}$; see Fig. 7. Data taken on February 4 were excluded in the analysis because of erroneous Doppler corrections.

$-2 \mathrm{I} /$ Borisov. Being only the second interstellar object known to have visited our Solar System, it was the first such object to show outgassing activity. This was confirmed in September 2019

\footnotetext{
https://wirtanen.astro.umd.edu/46P/46P_2018.shtml
} 

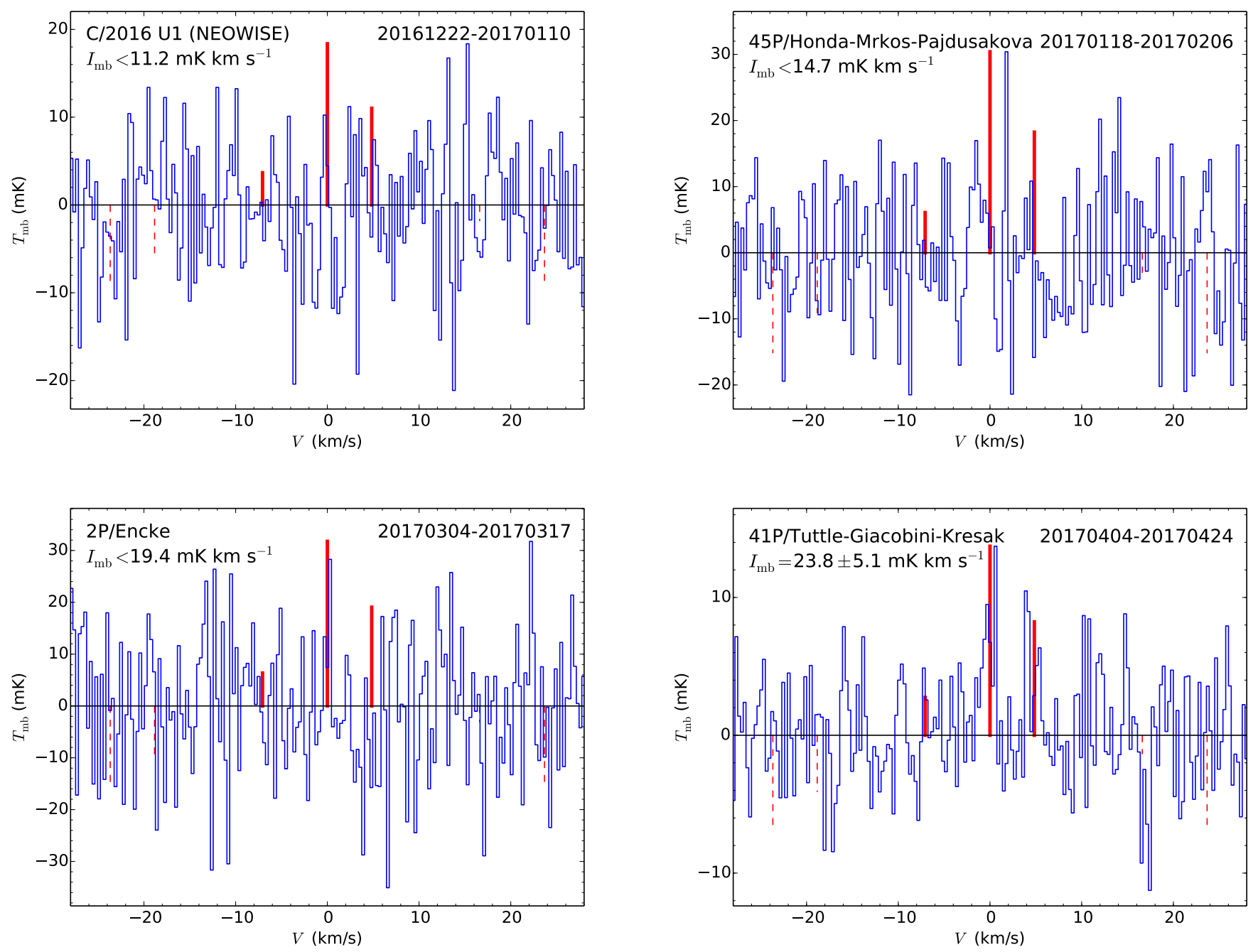

Fig. 1. OSO 20-m HCN 1-0 spectra towards the comets U1, 45P, 2P/Encke, and 41P. The velocity scale is in the reference frame of the comets and the intensity scale is in $T_{\mathrm{mb}}$. The positions of the three hfs components are marked with red bars. The relative heights of the bars correspond to the statistical weights, $g_{\mathrm{u}}$, in Table 1 . The integrated intensity is also shown together with $1 \sigma$ error or as $1 \sigma$ upper limit where applicable.

(Fitzsimmons et al. 2019). From interferometry imaging observations using ALMA (Cordiner et al. 2020) and Hubble Space Telescope (HST) observations (Bodewits et al. 2020), a COproduction rate of about $(5-10) \times 10^{26} \mathrm{~mol} \mathrm{~s}^{-1}$ around perihelion was found. Cordiner et al. (2020) also determined the HCN production rate to be $7 \times 10^{23} \mathrm{~mol} \mathrm{~s}^{-1}$. The $20-\mathrm{m}$ observations in October and November 2018 only resulted, when averaging all data, in an upper limit of the HCN 1-0 emission; see Fig. 7.

In the cases of 46P/Wirtanen (both $\mathrm{HCN}$ and $\mathrm{CH}_{3} \mathrm{OH}$ ) and C/2018 Y1 (Iwamoto), we see slightly shifted profiles in the sense that their peaks appear shifted with $\sim 0.3-0.4 \mathrm{~km} \mathrm{~s}^{-1}$ to lower velocities; see Figs. 4, 6, and 7. This apparent blueshift likely indicates that the gas outflow component directed towards Earth is more pronounced. As both these comets were at $R_{\mathrm{h}}>$ $1 \mathrm{au}$ at the time of our observations, much of their sunward sides were then facing Earth. To illustrate this asymmetry more clearly, a global average of all $\mathrm{HCN}$ high-resolution data for 46P/Wirtanen is also shown in Fig. 4 for which the blue peak is centred at $-0.4 \pm 0.1 \mathrm{~km} \mathrm{~s}^{-1}$ when fitted with a Gaussian. The ratio of the blue to red wing emission for this spectrum is $2.6 \pm 0.2$ when using the velocity ranges $\pm(0.15-0.85) \mathrm{km} \mathrm{s}^{-1}$ for the strongest $J_{F}=1_{2}-0_{1}$ line. The observed line widths for $46 \mathrm{P}$ and $\mathrm{Y} 1$ seem to indicate an expansion velocity of between 0.5 and $0.6 \mathrm{~km} \mathrm{~s}^{-1}$.

\section{Radiative transfer modelling of comets}

As mentioned above, water is the main ingredient in the volatile material that is being outgassed from the comet surface. The gaseous material leaves the comet surface with a nearly constant expansion velocity $v_{\mathrm{e}}$. Further out in the coma, the water molecules may be photoionized into $\mathrm{H}_{2} \mathrm{O}^{+}$and photodissociated mainly into $\mathrm{OH}$ and $\mathrm{H}$ (Rubin et al. 2009). Other molecules, like $\mathrm{HCN}$, behave in a similar way. In addition, $\mathrm{HCN}$ is an important precursor to CN (Fray et al. 2005; Paganini et al. 2010) although heated $\mathrm{CN}$-bearing dust could be a significant source of gaseous CN according to Hänni et al. (2020). This pure gas expansion may of course be modified when for example ions start to deflect when interacting with the solar wind. This happens near what is called the contact surface, $R_{\mathrm{CS}}$ (Rubin et al. 2009). However, the radial distribution of neutral parent molecules (i.e. those with origin from the comet surface) is thought to follow the Haser relation (Haser 1957):

$n_{\mathrm{mol}}(r)=\frac{Q_{\mathrm{mol}}}{4 \pi r^{2} v_{\mathrm{e}}} \exp \left(-r \gamma_{\mathrm{p}} / v_{\mathrm{e}}\right)$,

as a function of cometocentric radius $r$, where $Q_{\text {mol }}$ is the molecular production rate and $\gamma_{\mathrm{p}}$ is the photodissociation rate at the distance to the Sun. 

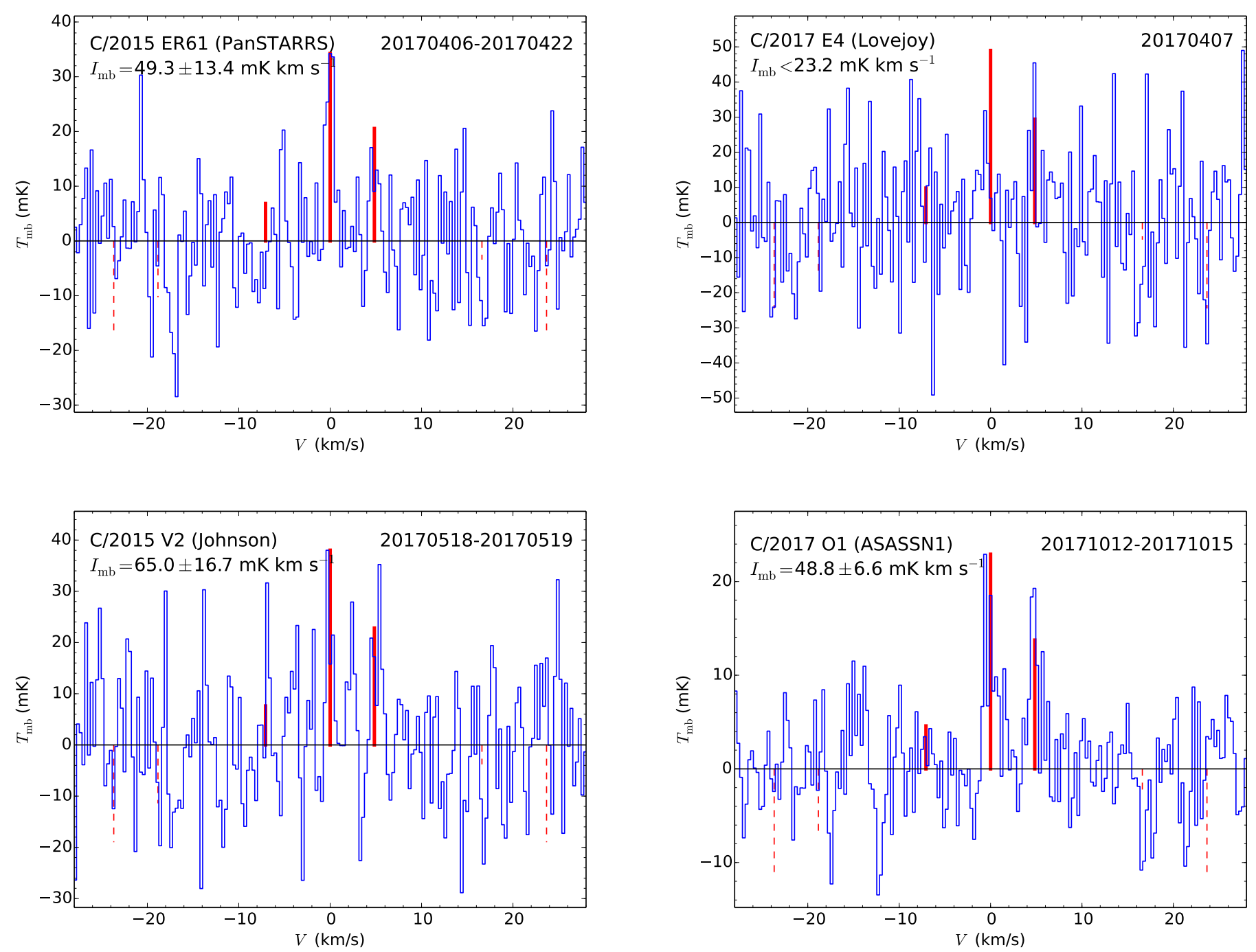

Fig. 2. HCN 1-0 spectra towards the comet ER61, E4, V2, and O1. Scales are as in Fig. 1.

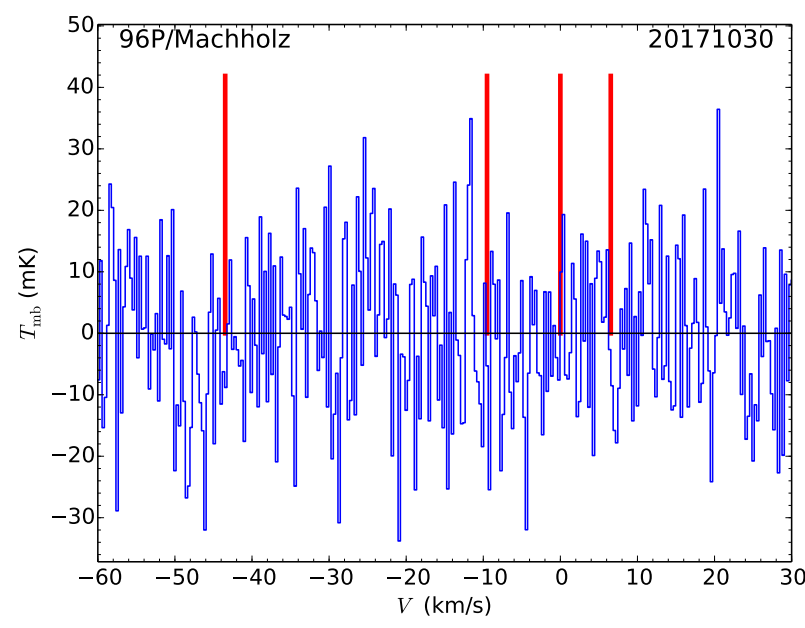

Fig. 3. $\mathrm{CH}_{3} \mathrm{OH}$ spectrum towards the comet $96 \mathrm{P} / \mathrm{Machholz}$ on 30 October 2017. The positions where the $\mathrm{CH}_{3} \mathrm{OH}$ lines would appear are indicated by the red bars. Scales are as in Fig. 1.

\subsection{Basic equations}

As pointed out by Crovisier \& Le Bourlot (1983), the change rate of the physical conditions, like density decline due to
Table 5. Observed $\mathrm{CH}_{3} \mathrm{OH}$ intensities and upper limit.

\begin{tabular}{lcc}
\hline \hline Comet & Line & $\begin{array}{c}I_{\mathrm{mb}} \\
\mathrm{mK} \mathrm{km} \mathrm{s}{ }^{-1}\end{array}$ \\
\hline 96P/Machholz & $J_{K}=2_{0}-1_{0} A^{+}$ & $<10.7$ \\
46P/Wirtanen & $J_{K}=2_{0}-1_{0} A^{+}$ & $34.3 \pm 2.7$ \\
& $J_{K}=2_{1}-1_{1} A^{+}$ & $20.0 \pm 3.1$ \\
& $J_{K}=8_{0}-7_{1} A^{+}$ & $28.3 \pm 2.7$ \\
& $J_{K}=2_{-1}-1_{-1} E$ & $19.6 \pm 2.7$ \\
& $J_{K}=2_{0}-1_{0} E$ & $19.0 \pm 2.7$ \\
& $J_{K}=2_{+1}-1_{+1} E$ & $13.4 \pm 2.7$ \\
\hline
\end{tabular}

expansion, in the cometary comae is not significantly different from the scale of molecular (in this case $\mathrm{CO}$ ) radiative and collisional rates, although their relative importance varies with radius. Here, we mainly follow the approach by Chin \& Weaver (1984), Bockelée-Morvan et al. (1984), Crovisier (1987), and Bockelée-Morvan (1987) when setting up the basic equations governing the excitation.

For simplicity, we start with a two-level system with upper level population $n_{\mathrm{u}}$ and lower level population $n_{1}$. The total population is $n=n_{\mathrm{u}}+n_{\mathrm{l}}$. The population change for the two levels 

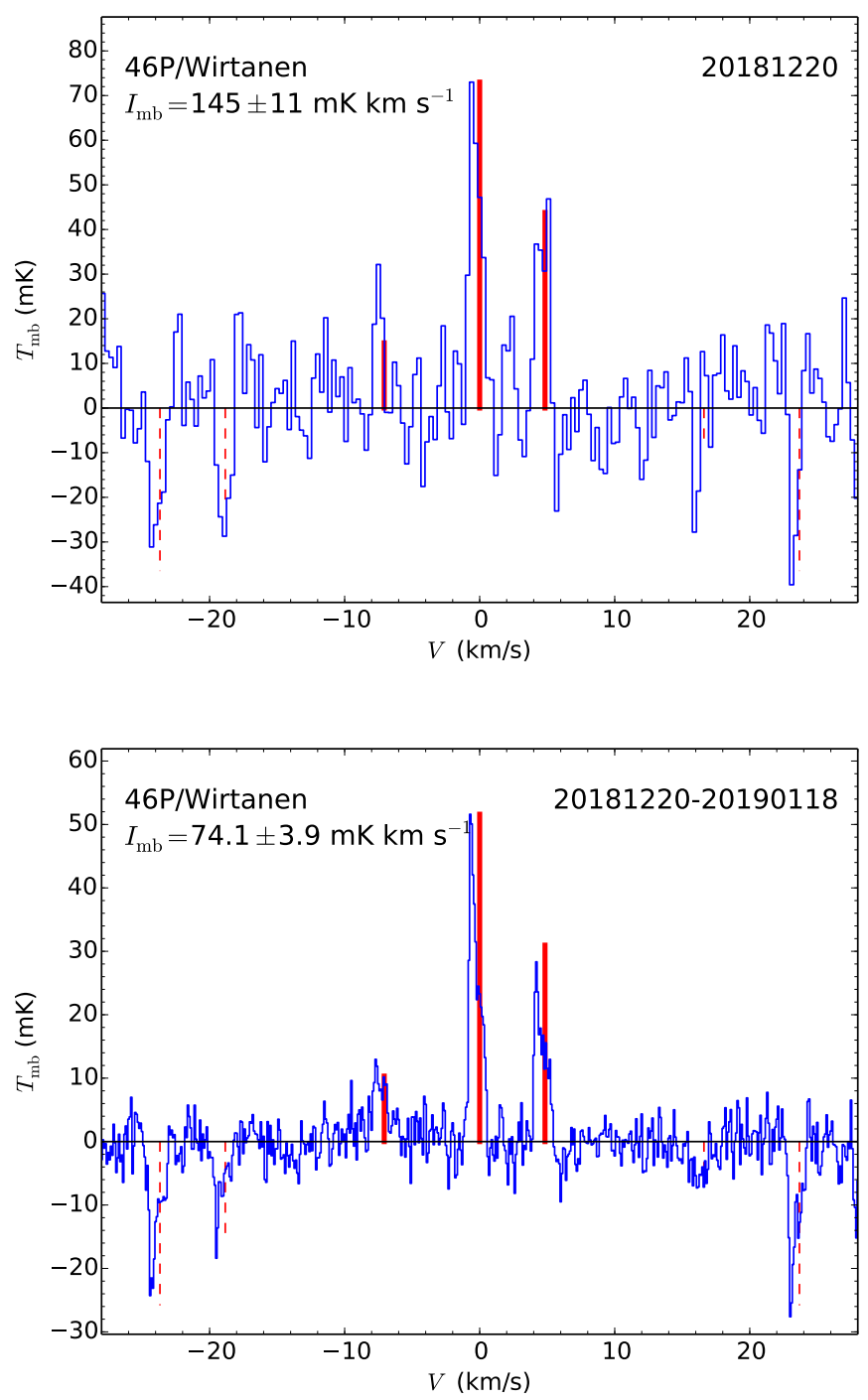

Fig. 4. Top: HCN 1-0 spectrum towards the comet 46P/Wirtanen on 20 December 2018. The positions, corresponding to a frequency throw of $7 \mathrm{MHz}$, of the negative artifacts of the $\mathrm{HCN}$ lines have been marked with negative dashed red lines. Bottom: global average of all highresolution $\left(0.1 \mathrm{~km} \mathrm{~s}^{-1}\right) \mathrm{HCN}$ data observed from 20 December 2018 to 18 January 2019. Scales are as in Fig. 1.

can be expressed as the differential equations (DEs):

$\mathrm{d} n_{\mathrm{u}} / \mathrm{d} t=-n_{\mathrm{u}}\left(A_{\mathrm{ul}}+B_{\mathrm{ul}} J_{v}+c_{\mathrm{ul}}+\gamma_{\mathrm{u}}\right)+n_{\mathrm{l}}\left(B_{\mathrm{lu}} J_{v}+c_{\mathrm{lu}}\right)$

$\mathrm{d} n_{\mathrm{l}} / \mathrm{d} t=+n_{\mathrm{u}}\left(A_{\mathrm{ul}}+B_{\mathrm{ul}} J_{v}+c_{\mathrm{ul}}\right)-n_{\mathrm{l}}\left(B_{\mathrm{lu}} J_{v}+c_{\mathrm{lu}}+\gamma_{l}\right)$,

where $A_{\mathrm{ul}}$ is the spontaneous decay from $u$ to $l$ and $B_{\mathrm{ul}} J_{v}$ and $B_{\mathrm{lu}} J_{v}$ are the rates due to stimulated emission and absorption, respectively, when exposed to the averaged radiation field $J_{v}$ at the frequency $v$. The downward and upward collisional rates are denoted $c_{\mathrm{ul}}$ and $c_{\mathrm{lu}}$, respectively. These are the standard processes included for a two-level system. We have also added two other destruction rates referred to as $\gamma_{u}$ and $\gamma_{l}$. These could for example represent destruction due to photodissociation or photoionization. We hereafter assume that the destruction rate is equal for the upper and lower levels, that is, we set $\gamma=\gamma_{\mathrm{u}}=\gamma_{\mathrm{l}}$. By adding Eqs. (2) and (3), we then obtain

$\mathrm{d} n / \mathrm{d} t=-\gamma n$,

which simply tells us that the total population $n(t)$ is exponentially decaying with time (if $\gamma>0$ ).
In the case of a cometary coma, due to the outgassing, the molecules expand with a velocity according to $v_{\mathrm{e}}$, which we assume to be constant here. The cometocentric radius, $r$, is then given as $r=r_{\mathrm{c}}+v_{\mathrm{e}} t$ as a function of time. The radius of the comet nucleus is $r_{\mathrm{c}}$. The destruction rate can then be modified to

$\gamma=\gamma_{\mathrm{p}}+\frac{2}{r} \mathrm{~d} r / \mathrm{d} t=\gamma_{\mathrm{p}}+\frac{2 v_{\mathrm{e}}}{r_{\mathrm{c}}+v_{\mathrm{e}} t}$

where $\gamma_{\mathrm{p}}$ is the destruction rate due to photodissociation (or photoionization) and the second term, $2 v_{\mathrm{e}} / r$, reflects the population dilution due to constant expansion. We note that the first part in Eq. (5) allows an expansion velocity varying with radius. However, hereafter we always assume a constant gas expansion velocity. By changing $\mathrm{d} t$ to $\mathrm{d} r / v_{\mathrm{e}}$ in Eqs. (2) and (3), it is straightforward to rewrite the population changes as a function of $r$ instead of $t$. For instance, Eq. (4) then becomes

$\mathrm{d} n / \mathrm{d} r=-\left(\gamma_{\mathrm{p}} / v_{\mathrm{e}}+2 / r\right) n$,

which solves into the Haser distribution, Eq. (1), when integrated. Hence, by adopting this approach we can study possible time-dependent effects of the individual level populations $n_{\mathrm{u}}$ and $n_{1}$ as a function of cometocentric radius by solving the DEs in Eqs. (2) and (3) while still maintaining an overall decline due to the Haser equation. This may be of particular interest near the contact surface $\left(R_{\mathrm{CS}}\right)$ where the electron properties vary very rapidly (they enter into the equations via $c_{\mathrm{ul}}$ and $c_{\mathrm{lu}}$ ).

The above two-level system of equations is easily generalised into a multi-level system, but in order to simplify the solution of the DEs we here neglect the contribution from the line itself when calculating the averaged radiation field, $\bar{J}_{v}$ at any given radial point in the coma. We assume that the contributions to $\bar{J}_{v}$ only come from the diluted black body radiations of the comet nuclei, the Sun, and the $2.7 \mathrm{~K}$ cosmic background, respectively. Here we use the geometric dilution factors for the comet nuclei, $\beta_{\mathrm{c}}(r)$, and that for the Sun, $\beta_{\odot}$. The remaining fraction of the $4 \pi$ sky background is assumed to be filled by the $T_{\mathrm{cmb}}=2.7 \mathrm{~K}$ cosmic background radiation. Neglecting effects due to shadowing, this can be expressed as

$\bar{J}_{v}=\beta_{\mathrm{c}}(r) J\left(T_{\mathrm{c}}\right)+\beta_{\odot} J\left(T_{\odot}\right)+\left[1-\beta_{\mathrm{c}}(r)-\beta_{\odot}\right] J\left(T_{\mathrm{cmb}}\right)$.

Here, $J(T)=1 /(\exp (h v / k T)-1)$, where $h$ and $k$ are Planck's and Boltzmann's constants, respectively. The radiation temperatures used are the surface temperature of the comet, $T_{\mathrm{c}}$, and for the Sun we use $T_{\odot}=5772 \mathrm{~K}$ (Hertel \& Schulz 2015). The geometric dilution factor for the Sun, $\beta_{\odot}$, at 1 au is about $5 \times 10^{-6}$ and at the surface of the comet we have that $\beta_{\mathrm{c}}\left(r_{\mathrm{c}}\right)=1 / 2$. We have not included thermal radiation from dust particles in the modelling presented here.

In order to solve the DEs, Eqs. (2) and (3), we adopt initial population values at $t=0$ and $r=r_{\mathrm{c}}$ that are appropriate to the production rate, $n(0)=Q /\left(4 \pi r_{\mathrm{c}}^{2} v_{\mathrm{e}}\right)$, and the initial excitation is entirely governed by the temperature of the comet surface, $T_{\mathrm{c}}$. Following Bensch \& Bergin (2004), we adopt a constant neutral gas kinetic temperature in the coma cloud set to the $T_{\mathrm{c}}$ value. We note that this is a simplification and some adiabatic cooling can take place (Biver et al. 2015). We use a fourth-order RungeKutta scheme (Abramowitz \& Stegun 1972) when solving the DEs, adapting the step size because of the radial gradients. The coma model cloud is assumed to be spherically symmetric. At any radius, we can also determine level populations in the statistical equilibrium (SE) case similar to Paganini et al. (2010) by only including radiative and collisional processes. 


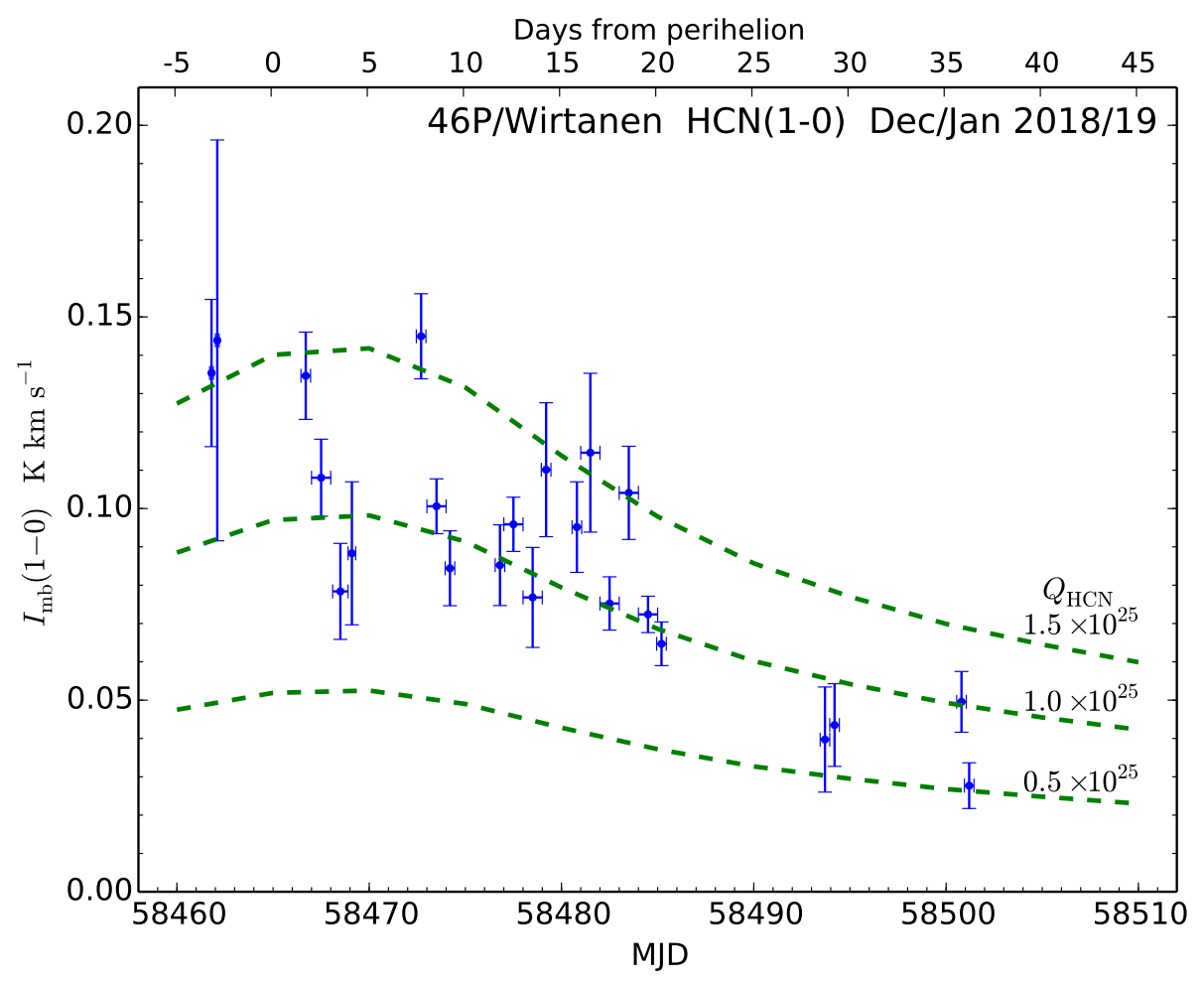

Fig. 5. OSO 20-m HCN 1-0 monitoring data towards 46P/Wirtanen in December 2018 and January 2019. The data points (blue marker with vertical $1 \sigma$ errors bars; horizontal bars indicate the range of observations), which are also listed in Table 4, represent the integrated intensity as a function of MJD or days from perihelion. The dashed lines represent the results from the radiative transfer modelling (see Sect. 4) using three different HCN production rates from $0.5 \times 10^{25} \mathrm{~mol} \mathrm{~s}^{-1}$ to $1.5 \times$ $10^{25} \mathrm{~mol} \mathrm{~s}^{-1}$ as indicated for a temperature of $70 \mathrm{~K}$ and $Q_{\mathrm{HCN}} / Q_{\mathrm{H}_{2} \mathrm{O}}=0.1 \%$.
We are only modelling $\mathrm{HCN}$ and $\mathrm{CH}_{3} \mathrm{OH}$ comet emission in this study. The overall photo-dissociation and photoionization rates for $\mathrm{HCN}, \mathrm{CH}_{3} \mathrm{OH}$, and $\mathrm{H}_{2} \mathrm{O}$ (given at 1 au from the quiet Sun) used come from the compilation by Huebner et al. (1992) which, for the molecules considered here, are essentially the same as those listed in the more recent work by Huebner \& Mukherjee (2015). Depending on the heliocentric distance $R_{\mathrm{h}}$ of the comet, these are scaled by $\left(1 / R_{\mathrm{h}}\right)^{2}$. Furthermore, we assume $R_{\mathrm{h}}$ to be constant during the solution.

To properly account for collisional excitation we include collisions by electrons and $\mathrm{H}_{2} \mathrm{O}$ molecules. While the moleculeelectron collisions are relatively easy to determine, collisions by water molecules are much more difficult to compute. For instance, it is only very recently that collisional rate coefficients for the collision systems $\mathrm{CO}-\mathrm{H}_{2} \mathrm{O}(J \leq 10)$ were determined accurately (Faure et al. 2020). In the case of HCN (with hyperfine structure) and $\mathrm{CH}_{3} \mathrm{OH}$, no such calculations exist yet. Limited $(J<8) \mathrm{HCN}-\mathrm{H}_{2} \mathrm{O}$ collisional rate coefficients have been determined by Dubernet \& Quintas-Sánchez (2019) which are being used here together with the $\mathrm{HCN}-\mathrm{He}$ rates of Dumouchel et al. (2010) for higher $J(J \geq 8)$. The latter rates were scaled to approximately match the $J<8 \mathrm{HCN}-\mathrm{H}_{2} \mathrm{O}$ rates before being concatenated to the Dubernet \& Quintas-Sánchez (2019) rates. For $\mathrm{CH}_{3} \mathrm{OH}$, we use the $\mathrm{CH}_{3} \mathrm{OH}-\mathrm{H}_{2}$ rates of Rabli \& Flower (2010) (for para $\mathrm{H}_{2}$ ). We treat the methanol $A$ - and $E$-species separately and do not include excited torsional states. The neutral collisional rates used are those valid for a kinetic temperature, $T_{\mathrm{k}}$, of $50 \mathrm{~K}$ and then simply scaled with $\left(T_{\mathrm{k}} / 50 \mathrm{~K}\right)^{1 / 2}$ for other temperatures. We use the tabulated downward collisional rates and calculate the corresponding upward rates from detailed balance. For HCN-e collisional rates, we adopt those determined by Faure et al. (2007) and for the electron excitation of $\mathrm{CH}_{3} \mathrm{OH}$ we use a set of rates computed in the Born approximation.

Normally, to speed up the calculations, we only include energy levels below $150-250 \mathrm{~K}$ for $\mathrm{HCN}$ and $\mathrm{CH}_{3} \mathrm{OH}$. The exact cut-off depends on the temperature. However, we have the option to include higher energy levels for testing if there are any truncation problems. Furthermore, vibrationally excited levels $\left(v_{2}\right)$ for HCN can also be fully included. However, the situation with a large number of levels together with levels with small populations requires much smaller step sizes to maintain the solution accuracy throughout the coma. This makes the computation very time consuming and a much faster way is to include effective pumping rates (see Paganini et al. (2010); Bensch \& Bergin (2004)), via the $v_{1}, v_{2}, 2 v_{2}$, and $v_{3}$ vibrational states. We only include the $P$ - and $R$-branches for the ro-vibrational $\mathrm{HCN}$ transitions here because they can cause redistribution of the ground-state level populations in the outer coma (e.g. BockeléeMorvan 1987). The $Q$-branch $(\Delta J=0)$ transitions do not do that by themselves (when neglecting rotational relaxations within the excited state). This way of incorporating effective pumping rates, adopting the radiation field in Eq. (7), is only valid when a small fraction of the molecules are in the excited states. This is typically the case for HCN (Bockelée-Morvan et al. 1984). We have not used any pumping rates for $\mathrm{CH}_{3} \mathrm{OH}$ in our modelling here.

To fully quantify the collisional processes as a function of cometocentric radius, not only the collisional rates are needed but also the radial distributions of water molecules and electrons. The water distribution is assumed to follow the Haser equation (Eq. (1)) assuming a water production rate $Q_{\mathrm{H}_{2} \mathrm{O}}$. The water production rate can be connected to the molecular ( $\mathrm{HCN}$ or $\mathrm{CH}_{3} \mathrm{OH}$ ) production rate $Q_{\text {mol }}$ via the abundance ratio $Q_{\text {mol }} / Q_{\mathrm{H}_{2} \mathrm{O}}$, which is normally referred to as the mixing ratio. To quantify the electron density radial distribution, $n_{\mathrm{e}}(r)$, we adopt the approach by Bensch \& Bergin (2004) who based their formulation on the work by Biver (1997) (see also Zakharov et al. 2007). Bensch \& Bergin (2004) express $n_{\mathrm{e}}(r)$ (and electron temperature) in terms of the water production rate and like Paganini et al. (2010) we use $x_{n_{\mathrm{e}}}=0.3$ as the density scaling factor. The rather complex radial behaviour of the electron density and temperature is visualised by Bensch \& Bergin (2004) in their Fig. 1. 

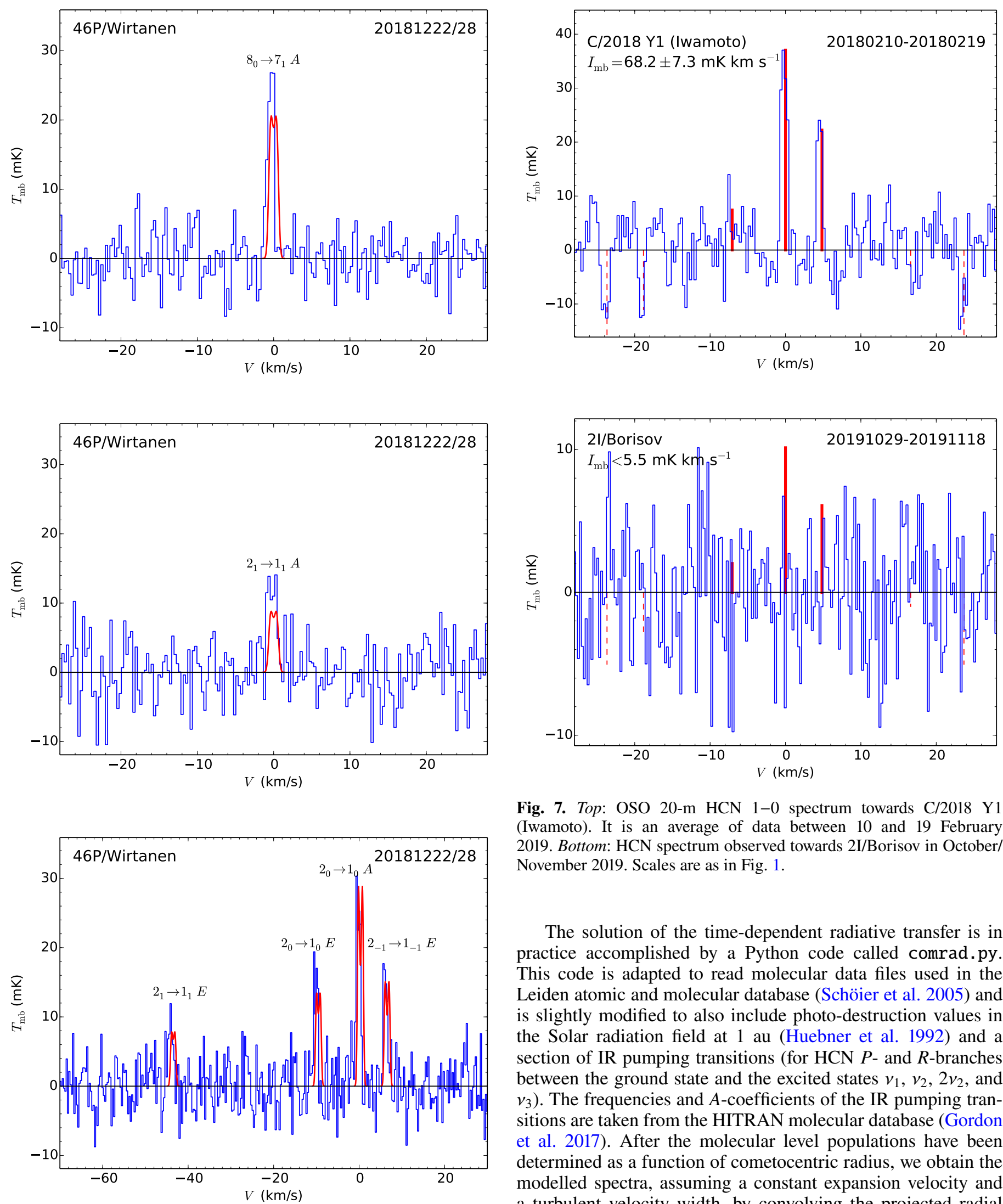

Fig. 6. OSO $20-\mathrm{m}$ methanol spectra towards $46 \mathrm{P} /$ Wirtanen when averaging data between 22 and 28 December 2018. The red spectra represent the modelling results of the radiative transfer modelling (see Sect. 4) using a $\mathrm{CH}_{3} \mathrm{OH}$ production rate of $1.6 \times 10^{26} \mathrm{~mol} \mathrm{~s}^{-1}$ and a kinetic temperature of $70 \mathrm{~K}$.

Fig. 7. Top: OSO $20-\mathrm{m}$ HCN $1-0$ spectrum towards $\mathrm{C} / 2018 \mathrm{Y} 1$ (Iwamoto). It is an average of data between 10 and 19 February 2019. Bottom: HCN spectrum observed towards 2I/Borisov in October/ November 2019. Scales are as in Fig. 1.

The solution of the time-dependent radiative transfer is in practice accomplished by a Python code called comrad.py. This code is adapted to read molecular data files used in the Leiden atomic and molecular database (Schöier et al. 2005) and is slightly modified to also include photo-destruction values in the Solar radiation field at 1 au (Huebner et al. 1992) and a section of IR pumping transitions (for $\mathrm{HCN} P$ - and $R$-branches between the ground state and the excited states $v_{1}, v_{2}, 2 v_{2}$, and $\left.v_{3}\right)$. The frequencies and $A$-coefficients of the IR pumping transitions are taken from the HITRAN molecular database (Gordon et al. 2017). After the molecular level populations have been determined as a function of cometocentric radius, we obtain the modelled spectra, assuming a constant expansion velocity and a turbulent velocity width, by convolving the projected radial intensity distribution with a Gaussian appropriate to the telescope beam size and the comet distance $(\Delta)$. Although the HCN level populations are strongly affected in the outer coma by the IR pumping transitions, the impact on the resulting line intensities of ground-state rotational transitions is small because the 
Table 6. Properties of the comets and results of the HCN modelling.

\begin{tabular}{lcccccccc}
\hline \hline Comet & $\begin{array}{c}R_{\mathrm{h}} \\
(\mathrm{au})\end{array}$ & $\begin{array}{c}\Delta \\
(\mathrm{au})\end{array}$ & $\begin{array}{c}r_{\mathrm{c}} \\
(\mathrm{km})\end{array}$ & $\begin{array}{c}v_{\mathrm{e}} \\
\left(\mathrm{km} \mathrm{s}^{-1}\right)\end{array}$ & $\begin{array}{c}T_{\mathrm{c}} \\
(\mathrm{K})\end{array}$ & $\begin{array}{c}Q_{\mathrm{H}_{2} \mathrm{O}} \\
\left(\mathrm{mol} \mathrm{s}^{-1}\right)\end{array}$ & $\begin{array}{c}Q_{\mathrm{HCN}} \\
\left(\mathrm{mol} \mathrm{s}^{-1}\right)\end{array}$ & $\begin{array}{c}Q_{\mathrm{HCN}} / Q_{\mathrm{H}_{2} \mathrm{O}} \\
(\%)\end{array}$ \\
\hline C/2016 U1 (NEOWISE) & 0.50 & 0.90 & 1.0 & 1.1 & 70 & $\ldots$ & $<7.8 \times 10^{25}$ & $\ldots$ \\
45P/Honda-Mrkos-Pajdušáková & 0.88 & 0.12 & $0.4^{(a)}$ & 0.8 & 70 & $2 \times 10^{27(b)}$ & $<3.2 \times 10^{24}$ & $<0.16$ \\
2P/Encke & 0.38 & 0.70 & $2.2^{(c)}$ & 1.3 & $70^{(d)}$ & $3 \times 10^{28(d)}$ & $<2.2 \times 10^{26}$ & $<0.7$ \\
41P/Tuttle-Giacobini-Kresák & 1.05 & 0.15 & $0.6^{(e)}$ & 0.8 & 70 & $3.5 \times 10^{27}(f)$ & $(4.5 \pm 1.0) \times 10^{24}$ & $0.13 \pm 0.03$ \\
C/2015 ER61 (PanSTARRS) & 1.10 & 1.22 & $0.4^{(c)}$ & 0.7 & $70^{(g)}$ & $1 \times 10^{29(g)}$ & $(8.2 \pm 2.1) \times 10^{25}$ & $0.082 \pm 0.021$ \\
C/2017 E4 (Lovejoy) & 0.63 & 0.67 & 1.0 & 1.0 & 70 & $3 \times 10^{28(h)}$ & $<6.3 \times 10^{25}$ & $<0.21$ \\
C/2015 V2 (Johnson) & 1.67 & 0.87 & $1.7^{(c)}$ & 0.6 & 60 & $4 \times 10^{28(i)}$ & $(3.5 \pm 0.9) \times 10^{25}$ & $0.088 \pm 0.022$ \\
46P/Wirtanen (2018-12-20) & 1.06 & 0.08 & $0.7^{(j)}$ & 0.55 & $70^{(k)}$ & $1.6 \times 10^{28(j)}$ & $(1.6 \pm 0.1) \times 10^{25}$ & $0.10 \pm 0.01$ \\
& & & & & & $8 \times 10^{27(l)}$ & $(1.5 \pm 0.1) \times 10^{25}$ & $0.19 \pm 0.02$ \\
C/2017 O1 (ASASSN1) & 1.50 & 0.72 & $0.92^{(c)}$ & 0.6 & 60 & $\ldots$ & $(2.2 \pm 0.2) \times 10^{25}$ & $\ldots$ \\
C/2018 Y1 (Iwamoto) & 1.30 & 0.42 & 1.0 & 0.55 & 60 & $2.1 \times 10^{28(m)}$ & $(2.2 \pm 0.2) \times 10^{25}$ & $0.10 \pm 0.02$ \\
2I/Borisov & 2.10 & 2.40 & 1.0 & 0.55 & $50^{(n)}$ & $6.5 \times 10^{26(n)}$ & $<6.3 \times 10^{24}$ & $<1.0$ \\
\hline
\end{tabular}

References. ${ }^{(a)}$ From Combi et al. (2019). ${ }^{(b)}$ From Dello Russo et al. (2020). ${ }^{\left({ }^{c}\right)}$ Comet radius from Paradowski (2020). ${ }^{(d)}$ From Roth et al. $(2018)$. ${ }^{\left({ }^{(e)}\right.}$ See Howell et al. (2017) and Boehnhardt et al. (2020). ${ }^{(f)}$ April 2017 average from Moulane et al. (2018). ${ }^{(g)}$ Adapted from Saki et al. (2021). ${ }^{(h)}$ From Faggi et al. (2018). ${ }^{(i)}$ From Combi et al. (2021). ${ }^{(j)}$ Adapted from Combi et al. (2020). ${ }^{(k)}$ Estimated from the $\mathrm{CH}_{3} \mathrm{OH}$ data in this paper. ${ }^{\left({ }^{(}\right)} \mathrm{Based}$ on IR spectroscopy data (Bonev et al. 2021). See also Lis et al. (2019). ${ }^{(m)}$ Preliminary data by DiSanti et al. (2019). ${ }^{(n)}$ Adapted from Cordiner et al. (2020).

major contribution comes from regions of high density (this can be pronounced by smaller observing beams).

In Appendix A we investigate the effects of the timedependent radiative transfer calculations by comparing the results to those of steady-state SE calculations. We use the timedependent radiative transfer model described above in the analysis of our $\mathrm{HCN}$ and $\mathrm{CH}_{3} \mathrm{OH}$ data and the results of the modelling are described in the following section.

\subsection{Model results}

Our HCN modelling results are summarised in Table 6. Here, the properties used for each comet (46P/Wirtanen only 20 December 2018) are also listed. The adopted nucleus radius, $r_{\mathrm{c}}$, which serves as a starting point for the model calculation, is also included but has essentially no impact on the modelling results. For $46 \mathrm{P}$ and $\mathrm{Y} 1$, we obtained the expansion velocities from the line widths. This results in $0.55 \mathrm{~km} \mathrm{~s}^{-1}$ for both comets. Coulson et al. (2020) found a similar value of $\sim 0.6 \mathrm{~km} \mathrm{~s}^{-1}$ for $46 \mathrm{P}$ ( at $R_{\mathrm{h}}=1.06 \mathrm{au}$ ). We note that, for 45P, Lovell et al. (2017) report an outflow velocity of about $0.8 \mathrm{~km} \mathrm{~s}^{-1}$ from $\mathrm{OH}$ observations (at $R_{\mathrm{h}}=0.54 \mathrm{au}$ ). Biver et al. (2006) determined a relation of $v_{\mathrm{e}}$ and heliocentric distance (in $\mathrm{au}$ ): $v_{\mathrm{e}} \sim 0.8 / \sqrt{R_{\mathrm{h}}} \mathrm{km} \mathrm{s}^{-1}$. This relation yields expansion velocities near $1.3 \mathrm{~km} \mathrm{~s}^{-1}$ for $2 \mathrm{P} /$ Encke and around $0.55 \mathrm{~km} \mathrm{~s}^{-1}$ for $2 \mathrm{I} /$ Borisov. The observed $v_{\mathrm{e}}$ around $0.55 \mathrm{~km} \mathrm{~s}^{-1}$ for $46 \mathrm{P}$ and $\mathrm{Y} 1$ lie below this relation. For $46 \mathrm{P}$ and $\mathrm{Y} 1$, the above relation predicts 0.76 and $0.70 \mathrm{~km} \mathrm{~s}^{-1}$, respectively. However, for the other comets we use an expansion velocity according to this relation.

In the case of $\mathrm{U} 1$ and $\mathrm{O} 1$, we cannot find representative water production rates in the literature. Here, we instead used the mean value of $Q_{\mathrm{HCN}} / Q_{\mathrm{H}_{2} \mathrm{O}}=0.1 \%$ from Bockelée-Morvan \& Biver (2017) to determine an appropriate $Q_{\mathrm{H}_{2} \mathrm{O}}$ for the modelling. It should be noted that the derived $Q_{\mathrm{HCN}}$ does not depend strongly on the adopted $Q_{\mathrm{H}_{2} \mathrm{O}}$ (as verified below by the $46 \mathrm{P}$ results where a factor of two increase in $Q_{\mathrm{H}_{2} \mathrm{O}}$ results in a $7 \%$ increase in the required $Q_{\mathrm{HCN}}$ ).

When possible we also use published values for the gas temperature (which is also used for the neutral gas kinetic temperature here and $T_{\mathrm{e}}$ out to $R_{\mathrm{CS}}$ ) as indicated in the table. The kinetic temperature is clearly dependent on the heliocentric distance $R_{\mathrm{h}}$ and Biver et al. (1997) estimated the kinetic temperature of $\mathrm{C} / 1995 \mathrm{O} 1$ (Hale-Bopp) from $\mathrm{CH}_{3} \mathrm{OH}$ (and $\mathrm{CO}$ ) observations as a function of $R_{\mathrm{h}}$ to be around $100 \mathrm{~K}$ at 1 au and approximately scaling as $R_{\mathrm{h}}^{-1}$ with heliocentric distance. In the case of 46P/Wirtanen, we use our $\mathrm{CH}_{3} \mathrm{OH}$ observations to estimate the kinetic temperature; see below. If a kinetic temperature estimate is lacking, we used $60 \mathrm{~K}$ for comets with $R_{\mathrm{h}} \geq 1.3 \mathrm{au}$ and $70 \mathrm{~K}$ for the remaining comets in the model calculations. These values approximately reflect the temperature behaviour as a function of heliocentric distance found by Biver et al. (1999) for C/1992 B2 (Hyakutake) or by DiSanti et al. (2016) in the case of D/2012 S1 (ISON). The temperature estimates used in Table 6 sometimes stem from rotational temperatures of molecules like $\mathrm{H}_{2} \mathrm{O}$ or $\mathrm{CH}_{3} \mathrm{OH}$. How well this rotational temperature reflects the gas kinetic temperature of the major collision agent $\left(\mathrm{H}_{2} \mathrm{O}\right)$ depends on the degree of thermalisation. Also, collisions by electrons may change the excitation of the molecular probe (Xie \& Mumma 1992).

Because of the observed blueshifted line profiles for 46P/Wirtanen and also C/2018 Y1 (Iwamoto), it is likely that there is an anisotropic distribution of $Q_{\mathrm{HCN}}$ in the sense that the production rate is higher on the sunward side of the comet than on the anti-sunward side (with a factor of 2-3 difference, see Fig. 4). This was also noted by Wang et al. (2020) and Biver et al. (2021) for $\mathrm{HCN}$ and for $\mathrm{CH}_{3} \mathrm{OH}$ by Roth et al. (2021c). Our modelled values, which are based on a spherically symmetric model geometry, therefore refer to an average production rate.

As mentioned above, in the case of 46P/Wirtanen, we can estimate the gas kinetic temperature because we detect lines with quite different upper energies (as high as $83 \mathrm{~K}$ ). Another aspect of $\mathrm{CH}_{3} \mathrm{OH}$ transitions, which is due to the nuclear spin directions of the methyl group $\mathrm{H}$ atoms, is that they come in two different symmetry species, $A$ and $E$, which are, as pointed out by Bockelée-Morvan et al. (1994), radiatively and collisionally uncoupled apart from possible line overlaps. The lowest $E$-state is about $8 \mathrm{~K}$ above the lowest $A$-state. Depending on the formation mechanism, they may be produced in unequal amounts. This 


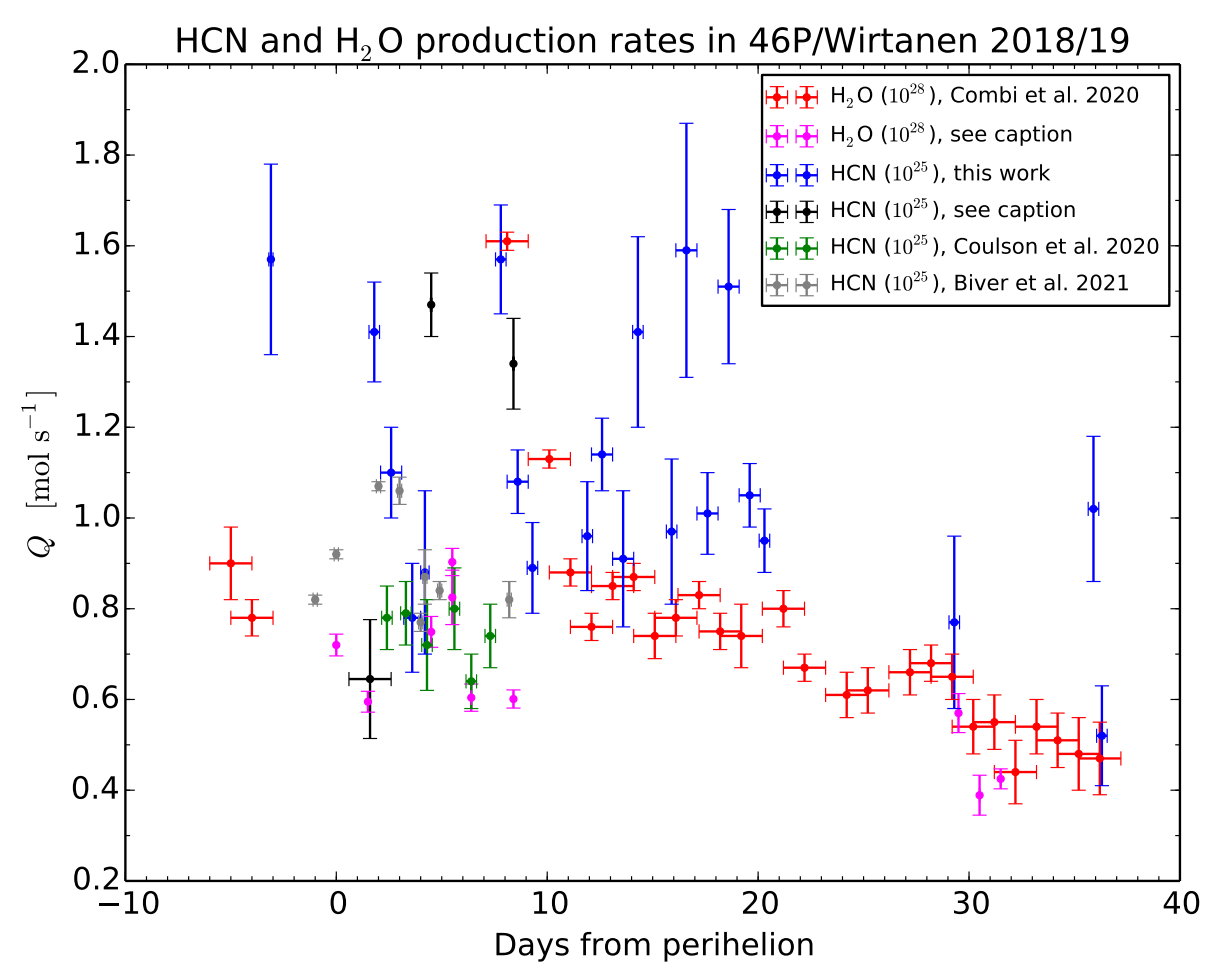

Fig. 8. $\mathrm{HCN}$ and $\mathrm{H}_{2} \mathrm{O}$ production rates in 46P/Wirtanen as a function of days from perihelion (12.9 December $2018 \mathrm{UT}$ ). The HCN rates (blue) were determined from the intensities (only those detected above the $3 \sigma$ level) in Table 4 . The water production rates (red) contemporary with our $Q_{\mathrm{HCN}}$ results, are from Combi et al. (2020). The perihelion value of $Q_{\mathrm{H}_{2} \mathrm{O}}$ from Moulane et al. (2019) and the post-perihelion values from Saki et al. (2020) (14 and 19 December), Roth et al. (2021a) (18 December), Bonev et al. (2021) (17/18 December), Khan et al. (2021) (21 December), and McKay et al. (2021) (11-13 January ) are included (magenta). Also, the $Q_{\mathrm{HCN}}$ as estimated by Wang et al. (2020) (14/15 December), Bonev et al. (2021) (17 December) and Khan et al. (2021) (21 December) are shown with black markers. The HCN observations by Coulson et al. (2020) have been included using green markers and those from Biver et al. (2021) are shown with grey markers. The lengths of the error bars in the vertical direction relate to the $1 \sigma$ uncertainties in the rates while the lengths in horizontal direction reflect the time length over which they refer to. As indicated, the HCN rates should be scaled by $10^{25}$ and the water rates by $10^{28}$.

can be the case at low temperatures (e.g. Wirström et al. 2011). If the temperature appropriate for methanol formation in comets is larger than $8 \mathrm{~K}$, then we expect the production rates of the $A$ - and $E$-species of $\mathrm{CH}_{3} \mathrm{OH}$ to be equal. We performed a grid testing with temperatures ranging from 40 to $120 \mathrm{~K}$ (in steps of $10 \mathrm{~K}$ ) and $Q_{\mathrm{CH}_{3} \mathrm{OH}}$ from $1.0 \times 10^{26} \mathrm{~mol} \mathrm{~s}^{-1}$ to $3.0 \times 10^{26} \mathrm{~mol} \mathrm{~s}^{-1}$ (in steps of $0.1 \times 10^{26} \mathrm{~mol} \mathrm{~s}^{-1}$ ). The water production rate used was $Q_{\mathrm{H}_{2} \mathrm{O}}=1.0 \times 10^{28} \mathrm{~mol} \mathrm{~s}^{-1}$ which is an average of the $Q_{\mathrm{H}_{2} \mathrm{O}}$ determined by Combi et al. (2020) for the dates 22-28 December 2018. The grid combination of kinetic temperature and $Q_{\mathrm{H}_{2} \mathrm{O}}$ that gave the best fit (as indicated by smallest $\chi^{2}$-value) to the observed integrated intensities in Table 5 was for a temperature of $70 \pm 15 \mathrm{~K}$ and $Q_{\mathrm{CH}_{3} \mathrm{OH}}=(1.6 \pm 0.1) \times 10^{26} \mathrm{~mol} \mathrm{~s}^{-1}$ or $1.6 \%$ of the water production rate.

The fitting also indicated, within the errors, that the $\mathrm{CH}_{3} \mathrm{OH}$ $A$ - and $E$-species are being outgassed in equal amounts in 46P/Wirtanen. The resulting model spectra are shown in Fig. 6 together with the observed spectra.

As noted above, the $\mathrm{CH}_{3} \mathrm{OH}$ modelling of 46P/Wirtanen resulted in a kinetic temperature of $70 \mathrm{~K}$. Using this temperature and $Q_{\mathrm{H}_{2} \mathrm{O}}=1.6 \times 10^{28} \mathrm{~mol} \mathrm{~s}^{-1}$ which is the 22 December value of Combi et al. (2020), we find that for the 20 December data an HCN production rate of $(1.6 \pm 0.1) \times 10^{25} \mathrm{~mol} \mathrm{~s}^{-1}$ matches the observed integrated intensity; see Tables 6 and 4. Using a fixed value for the ratio $Q_{\mathrm{HCN}} / Q_{\mathrm{H}_{2} \mathrm{O}}$ of $0.1 \%$, as found for the 20 December data, we ran models for three different $\mathrm{HCN}$ production rates at different dates and entered the expected 1-0 integrated intensity in Fig. 5. The production rates are $(0.5,1.0,1.5) \times 10^{25} \mathrm{~mol} \mathrm{~s}^{-1}$ which encompass most of the observed line intensities. Also included in Table 6 is the HCN production rate when adopting a lower water production rate as indicated by the infrared spectroscopy results (e.g. Bonev et al. 2021) a few days prior to 20 December. The change in $Q_{\mathrm{HCN}}$ is less than $10 \%$. The individual $\mathrm{HCN}$ production rates (see Table 4) are shown in Fig. 8 together with the water production rates obtained using SOHO/SWAN by Combi et al. (2020). These results are discussed in the following section.

Our $\mathrm{CH}_{3} \mathrm{OH}$ observations of $96 \mathrm{P} / \mathrm{Machholz}$ only resulted in an upper limit; see Table 5. Adopting $T_{\mathrm{c}}=90 \mathrm{~K}$ and $Q_{\mathrm{CH}_{3} \mathrm{OH}} / Q_{\mathrm{H}_{2} \mathrm{O}}=1 \%$, this upper limit corresponds to a very high methanol production rate. We estimate the upper limit production rate to be above $10^{28} \mathrm{~mol} \mathrm{~s}^{-1}$. The poor constraint of $Q_{\mathrm{CH}_{3} \mathrm{OH}}$ is due to the fact that $96 \mathrm{P}$ was only at $R_{\mathrm{h}}=0.12$ au at the time of the measurements and the size of the neutral coma is considerably smaller because of the effective destruction of $\mathrm{H}_{2} \mathrm{O}$ and $\mathrm{CH}_{3} \mathrm{OH}$ molecules near the Sun.

\section{Discussion}

In this section, we first discuss the previously determined HCN production rates as well as the upper limits. We also discuss the $\mathrm{HCN}$ production rates as an indirect indicator of water production rates via mixing ratios. In the case of 46P/Wirtanen, the monitoring results can be used to see variations in the HCN production rates on timescales of 1 day and these are compared to other observations of $\mathrm{HCN}$ and $\mathrm{H}_{2} \mathrm{O}$ production rates. Our usage 
of methanol as a thermal probe is also discussed. Finally, our time-dependent treatment of the radiative transfer of comets is discussed in relation to a more standard approach performed in the steady-state limit of the SE assumption.

\subsection{HCN production rates}

As can be seen in Table 6, we detected HCN in two JFCs (41P and 46P, the latter comet is discussed in more detail in the following section) and four long-period OCCs (ER61, V2, O1, and $\mathrm{Y} 1)$. For the six comets with $\mathrm{HCN}$ detections, the determined $\mathrm{HCN}$ production rates fall in the range $(0.5-8) \times 10^{25} \mathrm{~mol} \mathrm{~s}^{-1}$ and there is no obvious difference between the two types of comets. For the five comets where we also have independent (and reasonably contemporary) water production rates, the $\mathrm{HCN}$ to $\mathrm{H}_{2} \mathrm{O}$ mixing ratios are in the range $0.08-0.13 \%$. This range of mixing ratios is consistent with the values as determined from radio observations and discussed by Mumma \& Charnley (2011) and by Bockelée-Morvan \& Biver (2017). However it is smaller than the typical value in Dello Russo et al. (2016) by a factor of about two. The latter study, based on high-resolution infrared spectroscopy, compiles a typical range of mixing ratios of 0.15 $0.27 \%$. Our ratios, near $0.1 \%$ for $41 \mathrm{P}, \mathrm{ER} 61, \mathrm{~V} 2,46 \mathrm{P}$, and $\mathrm{Y} 1$, are outside this range but are well above the lower extreme value of $0.03 \%$ found by Dello Russo et al. (2016).

In the case of 41P, our $\mathrm{HCN}$ data were obtained over a longer period in April 2017. The average water production rate derived from $\mathrm{OH}$ observations by Moulane et al. (2018) over this period is estimated to be about $3.5 \times 10^{27} \mathrm{~mol} \mathrm{~s}^{-1}$ with no larger variations. Moulane et al. (2018) also report $\mathrm{CN}$ production rates in the range $(4-5) \times 10^{24} \mathrm{~mol} \mathrm{~s}^{-1}$ which is similar to our $\mathrm{HCN}$ production rate of $(4.5 \pm 1.0) \times 10^{24} \mathrm{~mol} \mathrm{~s}^{-1}$. This could indicate that the $\mathrm{CN}$ radicals observed in $41 \mathrm{P}$ mainly stem from photodissociation of HCN. However, given the uncertainties and that our $Q_{\mathrm{HCN}}$ is an average (over time and over the coma), one cannot exclude a contribution to $Q_{\mathrm{CN}}$ from dust grains as suggested by Dello Russo et al. (2016) and Hänni et al. (2020).

The ER61 observations ( $\mathrm{HCN}$ and $\mathrm{H}_{2} \mathrm{O}$ ) by Saki et al. (2021) occurred on three occasions in our 6-22 April observation range and here we adopt an average of their water production rates of $1 \times 10^{29} \mathrm{~mol} \mathrm{~s}^{-1}$. These authors deduced HCN mixing ratios in the range $0.11-0.14 \%$, somewhat higher than our value of $0.082 \%$. This is possibly due to the fact that our HCN data reflect a larger time-span. However, the ALMA results (Roth et al. 2021b) indicate an HCN mixing ratio $(0.072 \%)$ consistent with our value.

For Y1, the used water production rate of DiSanti et al. (2019) dates from about a week before our estimate of the $\mathrm{HCN}$ production rate. Adopting this $Q_{\mathrm{H}_{2} \mathrm{O}}$, the mixing ratio is $0.10 \pm 0.02 \%$. DiSanti et al. (2019) obtain an HCN mixing ratio of $0.2 \%$ on 4 February 2018. This could indicate variations in $Q_{\mathrm{H}_{2} \mathrm{O}}$ if we assume that the $\mathrm{HCN}$ production rate is constant over this period of observations.

In the case of the remaining $\mathrm{HCN}$ detections, $\mathrm{V} 2$ and $\mathrm{O} 1$, no other directly determined molecular production rates have been found to date in the literature. However, for V2, Combi et al. (2021) estimated the water production rate using SOHO/SWAN observations to about $4 \times 10^{28} \mathrm{~mol} \mathrm{~s}^{-1}$ near our observation date. The corresponding mixing ratio is about $0.08 \%$. We also note that Venkataramani \& Ganesh (2018) reported on the absence of molecular emissions towards V2 at $R_{\mathrm{h}}=2.83$ au but they noted that such emissions appeared later on a low level when $R_{\mathrm{h}}=$ $2.3 \mathrm{au}$. Our $3.9 \sigma$ detection was made at $R_{\mathrm{h}}=1.67 \mathrm{au}$.
In five of our observed comets (U1, 45P, 2P/Encke, E4, and 2I/Borisov), we only obtained upper limits on $Q_{\mathrm{HCN}}$; see Table 6. At the time of writing, no molecular observational results for U1 have been reported in the literature and so here we have nothing to compare to. The HCN production rate of $(3-4) \times 10^{24} \mathrm{~mol} \mathrm{~s}^{-1}$ in 45P found by Dello Russo et al. (2020) refers to dates in midFebruary 2017. Our non-detection, $Q_{\mathrm{HCN}}<3.2 \times 10^{24} \mathrm{~mol} \mathrm{~s}^{-1}$, is from early February and indicates that the production rate of HCN was about the same or lower in early February. Roth et al. (2018) observed $\mathrm{HCN}$ in $2 \mathrm{P} /$ Encke after perihelion on 21 and 25 March, resulting in $Q_{\mathrm{HCN}} \sim(3-6) \times 10^{25} \mathrm{~mol} \mathrm{~s}^{-1}$. Our upper limit only indicates that $Q_{\mathrm{HCN}}$, before and around perihelion, did not exceed 6-8 times this value. For E4, Faggi et al. (2018) determined an HCN production rate of $5 \times 10^{25} \mathrm{~mol} \mathrm{~s}^{-1}$. This was 3 days before we obtained an upper limit of $6 \times$ $10^{25} \mathrm{~mol} \mathrm{~s}^{-1}$ and so apparently there was no significant increase in $Q_{\mathrm{HCN}}$ from April 4 to 7 . Our upper limit on $Q_{\mathrm{HCN}}$ towards 2I/Borisov is consistent with the ALMA HCN detection on 14 and 15 December by Cordiner et al. (2020). They determined that $Q_{\mathrm{HCN}}=7 \times 10^{23} \mathrm{~mol} \mathrm{~s}^{-1}$. Taken together, based on our $J=1-0$ data, the $\mathrm{HCN}$ mixing ratios seem to fall near $0.1 \%$ when we have reasonably contemporary estimates of $Q_{\mathrm{H}_{2} \mathrm{O}}$.

\subsection{Variation in the $H C N$ production rates for $46 P /$ Wirtanen}

We determined $\mathrm{HCN}$ production rates for 46P/Wirtanen (Table 4) from a few days before perihelion until 36 days after perihelion; see Figs. 5 and 8. There are clear variations in $Q_{\mathrm{HCN}}$, sometimes on a daily basis, but it is also evident that $Q_{\mathrm{HCN}}$ has decreased by a factor of 2-3 some month after the perihelion passage. We note that Wang et al. (2020) observed HCN 1-0 on 14-15 December and obtained a $Q_{\mathrm{HCN}}$ (based on LTE calculations) of about half our value and that the IRAM 30-m observations (Biver et al. 2021) on 14 December result in a $Q_{\mathrm{HCN}}$ in between our value $\left(1.4 \times 10^{25} \mathrm{~mol} \mathrm{~s}^{-1}\right)$ and that of Wang et al. (2020). A few days later, in the range 15-17 December, the IRAM $30-\mathrm{m}$ data show $Q_{\mathrm{HCN}}$ determinations very close to our values. The James Clerk Maxwell Telescope (JCMT) HCN(43) data of Coulson et al. (2020) showed little daily variation in $Q_{\mathrm{HCN}}$ in the period 15 December to the early UT hours of 20 December. The HCN production rates determined by Wang et al. (2020), Biver et al. (2021) and Coulson et al. (2020) are all included in Fig. 8. The $\mathrm{HCN}(4-3) Q_{\mathrm{HCN}}$ results agree relatively well with our results up until 20 December. Here we see a clear increase in $Q_{\mathrm{HCN}}$ to $(1.6 \pm 0.1) \times 10^{25} \mathrm{~mol} \mathrm{~s}^{-1}$ by a factor of two over that from Coulson et al. (2020). However, the JCMT observations relate to the early UT hours and our observations are from about $12 \mathrm{~h}$ later on the same day. This would indicate an outburst event during the day of 20 December. However, early on 21 December the zero-spacing NOEMA observations of Biver et al. (2021) again indicate a lower $Q_{\mathrm{HCN}}$ of $0.8 \times 10^{25} \mathrm{~mol} \mathrm{~s}^{-1}$, meaning that if there was an outburst it must have been rather short ( $\$ 12 \mathrm{~h}$ ) so as not to be recorded by the NOEMA observations. In fact, similar changes of $Q_{\mathrm{CH}_{3} \mathrm{OH}}$ by about a factor of two were seen by Roth et al. (2021c), connecting it to the rotation period timescale of $9 \mathrm{~h}$.

Based on infrared spectroscopy, Bonev et al. (2021) reported an $\mathrm{HCN}$ production rate of $(1.47 \pm 0.07) \times 10^{25} \mathrm{~mol} \mathrm{~s}^{-1}$ on 17 December. This $Q_{\mathrm{HCN}}$ is significantly higher than our value of $(0.9 \pm 0.2) \times 10^{25} \mathrm{~mol} \mathrm{~s}^{-1}$ and the value $(0.7 \pm 0.1) \times 10^{25} \mathrm{~mol} \mathrm{~s}^{-1}$ of Coulson et al. (2020) from the same date. Four days later, on 21 December, Khan et al. (2021) observed HCN, also by infrared spectroscopy. Their HCN production rate averaged to about $1.3 \times 10^{25} \mathrm{~mol} \mathrm{~s}^{-1}$ which is near our value of $1.1 \times 10^{25} \mathrm{~mol} \mathrm{~s}^{-1}$ 
(see Table 4) on the same date. The $Q_{\mathrm{HCN}}$ values from Bonev et al. (2021) and Khan et al. (2021) have been included in Fig. 8.

As already pointed out in Sect. 4.2, there appears to be a clear difference of a factor of two to three in the outgassing activity on the sunward versus anti-sunward parts of the comet nucleus over the course of about one month. As reported by Biver et al. (2015), based on their water line observations by the Microwave Instrument for the Rosetta Orbiter (MIRO) of the JFC 67P/Churyumov-Gerasimenko, the nightside water production rate was low, $<1 \%$ of the dayside production rate (at $r_{\mathrm{h}}=3.4 \mathrm{au}$ ). Later, at $r_{\mathrm{h}}=1.8 \mathrm{au}$, Fink et al. (2016) report for $67 \mathrm{P}$ that about $17 \%$ of the total water production emerges from the nightside. In any case, this is a higher day-to-nightside activity ratio than we see for $46 \mathrm{P}$ but our ratio is averaged over a period of a month in which the daytime fraction as seen by our telescope beam changed (elongation varied from 160 to $140 \mathrm{deg}$ from 20 December 2018 to 18 January 2019).

The SOHO/SWAN monitoring observations (Combi et al. 2020 ) to estimate $Q_{\mathrm{H}_{2} \mathrm{O}}$ in $46 \mathrm{P}$ coincide partly in time with our observations and provide estimates of the water production rate variation. As these results are based on Ly- $\alpha$ observations of the hydrogen coma (dissociation product from $\mathrm{H}_{2} \mathrm{O}$ and $\mathrm{OH}$ ), they refer to an average water production of the 2-3 days prior to the actual observation date (as indicated by the horizontal error bars in Fig. 8) and so the $Q_{\mathrm{H}_{2} \mathrm{O}}$ determined this way may not probe activity variations on shorter timescales. However, the trend we see in $Q_{\mathrm{HCN}}$ from 20 December until 18 January (836 days after perihelion) is consistent with a mixing ratio near $0.1 \%$. For the week preceding this time-span, Biver et al. (2021) report almost the same value $0.11 \pm 0.01 \%$. There are a few $Q_{\mathrm{HCN}}$ estimates in our monitoring time-span 8-36 days after perihelion that seem to indicate an $\mathrm{HCN}$ mixing ratio near $0.2 \%$. This is also the case for the pre-perihelion observations. To distinguish whether this reflects real changes in the mixing ratio by a factor of about two or is an effect of different time-averaging would require $Q_{\mathrm{H}_{2} \mathrm{O}}$ measurements with $\sim 1$ day time-resolution or better. If it is an effect of time-averaging, the $\mathrm{HCN}$ production rate will be a good indicator of the water production rate in the case of $46 \mathrm{P} /$ Wirtanen. The increase in $Q_{\mathrm{HCN}}$ seen by us on 20 December seems supported by the large water production rate reported by Combi et al. (2020) 2-3 days later and, as discussed earlier, the $Q_{\mathrm{HCN}}$ reported by Khan et al. (2021) on 21 December but not by the NOEMA observations in the early UT hours on 21 December.

\subsection{Coma gas kinetic temperature and methanol production in 46P/Wirtanen}

In Sect. 4.2, we determined the gas kinetic temperature of $46 \mathrm{P}$ from methanol data taken over the time-span 22-28 December to be $70 \pm 15 \mathrm{~K}$. Furthermore, the deduced production rate of $Q_{\mathrm{CH}_{3} \mathrm{OH}}=(1.6 \pm 0.1) \times 10^{26} \mathrm{~mol} \mathrm{~s}^{-1}$ corresponds to a methanol mixing ratio of about $1.6 \%$. Our mixing ratio is very near the JFC average value of $1.7 \%$ as compiled by Dello Russo et al. (2016) and slightly less than the JFC median value of about $2 \%$ as compiled by Mumma \& Charnley (2011).

Most other methanol observations of $46 \mathrm{P}$ refer to the period around the perihelion or the week after. For instance, on 79 December, Roth et al. (2021c) used ALMA observations to determine methanol rotation temperatures in the range from 50 to $80 \mathrm{~K}$. Using IRAM $30-\mathrm{m} \mathrm{CH}_{3} \mathrm{OH}$ observations, Biver et al. (2021) determined six-day averages (over the range 1218 December) of the gas temperature in the range $53-75 \mathrm{~K}$. On 16 December, Coulson et al. (2020) used the JCMT to observe three methanol lines around $338 \mathrm{GHz}$. They determined the rotation temperature to $T_{\text {rot }} \sim 30-50 \mathrm{~K}$. Using infrared spectroscopy observations on 18 December, Roth et al. (2021a) report a methanol rotation temperature of about $90 \mathrm{~K}$. In addition, Biver et al. (2021) also made a single methanol observation on 25.8 December 2018 UT - within our observing time range -, which resulted in a gas temperature of $43 \pm 7 \mathrm{~K}$.

Three studies estimate $\mathrm{H}_{2} \mathrm{O}$ rotation temperatures of 46P. On 14 and 19 December, Saki et al. (2020) determined the water rotation temperature to be about $85 \mathrm{~K}$. On 18 December, Roth et al. (2021a) report a rotation temperature of about $90 \mathrm{~K}$. Later, on 21 December Khan et al. (2021) derived temperatures of $80 \mathrm{~K}$ to $90 \mathrm{~K}$. Our derived value of the gas kinetic temperature of about $70 \mathrm{~K}$, as averaged over 22-28 December, is consistent with the studies mentioned above. There is a possibility that the temperature decreased from $80-90 \mathrm{~K}$ to about 40-50 K during our observations (cf. Khan et al. 2021; Biver et al. 2021). However, this notion is somewhat complicated by the variation of temperature estimates (30-90 K) prior to our observations.

In the case of the methanol production in 46P, we begin with noting that on 7-9 December, the ALMA $\mathrm{CH}_{3} \mathrm{OH}$ observations (Roth et al. 2021c) resulted in varying $\mathrm{CH}_{3} \mathrm{OH}$ production rates in the range $(2.0-3.6) \times 10^{26} \mathrm{~mol} \mathrm{~s}^{-1}$. The IRAM $30-\mathrm{m}$ data by Biver et al. (2021) determined $Q_{\mathrm{CH}_{3} \mathrm{OH}}$ to $(2.6 \pm 0.2) \times$ $10^{26} \mathrm{~mol} \mathrm{~s}^{-1}$ averaged over $12-18$ December. The JCMT observations on 16 December (Coulson et al. 2020) resulted in $(3.5 \pm$ $0.2) \times 10^{26} \mathrm{~mol} \mathrm{~s}^{-1}$. On 18 December, Roth et al. (2021a) report a methanol production rate around $2 \times 10^{26} \mathrm{~mol} \mathrm{~s}^{-1}$. A few days later, on 21 December, Khan et al. (2021) reported a similar methanol production rate of $2.5 \times 10^{26} \mathrm{~mol} \mathrm{~s}^{-1}$. These estimates of $Q_{\mathrm{CH}_{3} \mathrm{OH}}$ are all higher than our later, 22-28 December value of $Q_{\mathrm{CH}_{3} \mathrm{OH}}=(1.6 \pm 0.1) \times 10^{26} \mathrm{~mol} \mathrm{~s}^{-1}$. The NOEMA observations (Biver et al. 2021) on 25.8 December 2018 UT resulted in $(1.84 \pm 0.14) \times 10^{26} \mathrm{~mol} \mathrm{~s}^{-1}$, which is close to our value.

As noted above, we adopt $Q_{\mathrm{H}_{2} \mathrm{O}}=1.0 \times 10^{28} \mathrm{~mol} \mathrm{~s}^{-1}$ which is an average of the $Q_{\mathrm{H}_{2} \mathrm{O}}$ determined by Combi et al. (2020) for the dates 22-28 December 2018. This $Q_{\mathrm{H}_{2} \mathrm{O}}$ results in a mixing ratio of $1.6 \%$. For the two-week period prior to our methanol observations, mixing ratios in the range $2-5 \%$ were reported (Roth et al. 2021c; Biver et al. 2021; Coulson et al. 2020; Roth et al. 2021a; Khan et al. 2021). Based on this, we suggest that the average methanol mixing ratio decreased by about a factor of two from around perihelion into our observing period of 22-28 December.

The production rates of the methanol $A$ - and $E$-species are about equal for $46 \mathrm{P}$ within the uncertainties. This would suggest a temperature environment of $\gtrsim 8 \mathrm{~K}$ during the formation of the methanol molecules. Finally, the determined kinetic temperature is related to the used $\mathrm{CH}_{3} \mathrm{OH}-\mathrm{H}_{2} \mathrm{O}$ collisional rate coefficients which in our case were based on the $\mathrm{CH}_{3} \mathrm{OH}-\mathrm{H}_{2}$ rates. As already pointed out, accurate collisional rate coefficients currently only exist for the collision systems $\mathrm{CO}-\mathrm{H}_{2} \mathrm{O}$ (Faure et al. 2020) and $\mathrm{HCN}-\mathrm{H}_{2} \mathrm{O}$ (Dubernet \& Quintas-Sánchez 2019).

\subsection{Time-dependent radiative transfer versus SE calculations}

To study time-dependent effects, we used fragment B of 73P as a test case (see Appendix A) and we noted that the HCN level populations deviated by $5-15 \%$ (for $J=0-5$ ) when comparing the time-dependent results with the steady-state SE-calculations. The water production rate for $73 \mathrm{P} / \mathrm{B}$ is around $2 \times 10^{28} \mathrm{~mol} \mathrm{~s}^{-1}$. We also found that the time-dependent deviations would be larger for comets with lower water production rates, because 
the collisions with water molecules will be less frequent in the inner part of the coma. Likewise, by reducing the effect of electron-HCN collisions, the time-dependent effects became more pronounced. For molecules with lower electric dipole moments (and hence generally lower $A$-coefficients), we expect the effects to be more pronounced, at least in the outer coma where radiative processes dominate (see Fig. A.1). However, strong IR-pumping could counteract the effect in the outer coma, making the details of the excitation picture very complicated. We expect the influence of time-dependent effects on the $\mathrm{HCN}$ level populations to be small ( $\$ 20 \%)$ for $Q_{\mathrm{H}_{2} \mathrm{O}} \gtrsim 10^{28} \mathrm{~mol} \mathrm{~s}^{-1}$.

It is worth pointing out that the total molecular population is bound to follow the radial Haser distribution so that molecular production rates based on the analysis of a larger number of transitions should be less affected by the time-dependent effects encountered here. Also, the present modelling work neglects excitation effects at a certain position in the coma caused by line radiation from other parts of the coma (same as assuming optically thin radiation in the level population determination). This may be a good approximation for most cometary molecular probes but an important exception is water excitation (e.g. Bockelée-Morvan 1987; Bensch \& Bergin 2004). Extending our model to using an escape probability formalism (cf. Gersch \& A'Hearn 2014) is a possibility, but would require an iterative solution technique; that is, solving for the entire coma using an initial guess (using the DE here or simply an SE solution), including the line radiation in Eq. (7), and then repeating the procedure with time-dependent solutions throughout the coma until the level populations converge. Presumably, this would be a very time-consuming technique but could be tried for water excitation in comets. Solution methods based on the Monte Carlo technique (Bernes 1979) or those using accelerated lambda iteration (Rybicki \& Hummer 1991; Bergman \& Humphreys 2020) appear to be less suitable for time-dependent studies because they rely on the SE steady state assumption. However, in the escape probability formalism (e.g. Bockelée-Morvan 1987; van der Tak et al. 2007; Zakharov et al. 2007), the $A$-coefficients are replaced by $\beta A$ where $\beta$ is the (local) escape probability for the transition in question. In the case of optically thick transitions $(\beta \sim 1 / \tau)$, this could imply that time-dependent effects due to a reduction in radiative excitation could be of greater importance.

Another aspect is that, because the radiative transfer formulation here is in the time domain, it would be easier to incorporate chemical and photolytic state-to-state reactions when solving excitation of several molecules at the same time in the coma. Finally, it is worth stressing that most radiative transfer models, including the one presented here, assume a spherically symmetric coma geometry. Remote observations and in situ studies of cometary comae clearly show deviations from this geometry and models like that of Debout et al. (2016) could be used for studying time-dependent effects also in anisotropic comae.

\section{Summary and conclusions}

We performed spectral line observations of $\mathrm{HCN}$ and $\mathrm{CH}_{3} \mathrm{OH}$ transitions towards a sample of bright comets using the OSO 20-m telescope. From the observations, we determined molecular production rates using a radiative transfer model taking into account time-dependent effects. Our main conclusions can be summarised as follows.

- We detected $\mathrm{HCN}(1-0)$ in six comets of our sample: two JFCs and four OCCs. For five of these comets, we were able to determine the $\mathrm{HCN}$ mixing ratio using published values of $Q_{\mathrm{H}_{2} \mathrm{O}}$. The determined HCN mixing ratios were all near $0.1 \%(0.08-0.013 \%)$

- We detected HCN emission in 46P/Wirtanen on 21 occasions from 9 December 2018 to 18 January 2019. When comparing with contemporary $Q_{\mathrm{H}_{2} \mathrm{O}}$ determinations, we find a typical mixing ratio near $0.1 \%$ with possible variations of up to $0.2 \%$. This variation can also be due to short-time activity variations ( $\lesssim 1 \mathrm{~d})$ not reflected in the Ly- $\alpha$ observations used for determining $Q_{\mathrm{H}_{2} \mathrm{O}}$. We also noted a clear asymmetry in the observed $\mathrm{HCN}$ and $\mathrm{CH}_{3} \mathrm{OH}$ line profiles in the sense that the sunward activity was about a factor of two to three stronger than the outgassing activity on the 46P night-side.

- In addition to $\mathrm{HCN}$, we detected six $\mathrm{CH}_{3} \mathrm{OH}$ transitions in 46P/Wirtanen around two weeks after perihelion in December 2018. This enabled us to determine the gas kinetic temperature to be about $70 \mathrm{~K}$. The methanol mixing ratio was found to be $1.6 \%$, which is about half that of the methanol mixing ratio during the two weeks prior to our observations.

- To interpret our $\mathrm{HCN}$ and $\mathrm{CH}_{3} \mathrm{OH}$ observations, we used a radiative transfer code in which we allowed for timedependent effects on the level populations by relaxing the normal steady-state assumption of statistical equilbrium (SE) in the cometary coma. In our test case (73P fragment B, see Fig. A.2) we noticed 5-15\% deviations of the HCN level populations as compared to SE values. We recognized that the time-dependent effects may be more pronounced for comets with lower water production rates and if the molecular excitation via electron collisions becomes less efficient. For $Q_{\mathrm{H}_{2} \mathrm{O}} \gtrsim 10^{28} \mathrm{~mol} \mathrm{~s}^{-1}$, the time-dependent effects in the HCN excitation should be small.

Acknowledgements. We are very grateful to L. Paganini for sharing his $\mathrm{HCN}$ modelling details. We also thank N. Biver for making his 1997 thesis available to us. The Onsala Space Observatory national research infrastructure is funded through Swedish Research Council grant No 2017-00648.

\section{References}

Abramowitz, M., \& Stegun, I. A. 1972, Handbook of Mathematical Functions (New York: Dover)

Belitsky, V., Lapkin, I., Fredrixon, M., et al. 2015, A\&A, 580, A29

Bensch, F., \& Bergin, E. A. 2004, ApJ, 615, 531

Bergman, P., \& Humphreys, E. M. L. 2020, A\&A, 638, A19

Bernes, C. 1979, A\&A, 73, 67

Biver, N. 1997, PhD thesis, Université Paris 7, France

Biver, N., Bockelée-Morvan, D., Colom, P., et al. 1997, Earth Moon Planets, 78 5

Biver, N., Bockelée-Morvan, D., Crovisier, J., et al. 1999, AJ, 118, 1850

Biver, N., Bockelée-Morvan, D., Crovisier, J., et al. 2006, A\&A, 449, 1255

Biver, N., Hofstadter, M., Gulkis, S., et al. 2015, A\&A, 583, A3

Biver, N., Bockelée-Morvan, D., Boissier, J., et al. 2021, A\&A, 648, A49

Bockelée-Morvan, D. 1987, A\&A, 181, 169

Bockelée-Morvan, D., \& Biver, N. 2017, Phil. Trans. R. Soc. London, Ser. A, 375,20160252

Bockelée-Morvan, D., Crovisier, J., Baudry, A., et al. 1984, A\&A, 141, 411

Bockelée-Morvan, D., Crovisier, J., Colom, P., \& Despois, D. 1994, A\&A, 287, 647

Bodewits, D., Noonan, J. W., Feldman, P. D., et al. 2020, Nat. Astron., 4, 867

Boehnhardt, H., Riffeser, A., Ries, C., Schmidt, M., \& Hopp, U. 2020, A\&A, 638, A8

Bonev, B. P., Mumma, M. J., DiSanti, M. A., et al. 2006, ApJ, 653, 774

Bonev, B. P., Dello Russo, N., DiSanti, M. A., et al. 2021, Planet. Sci. J., 2, 45

Brinkman, C. L. 2020, AAS Meeting Abs., 454.02

Chin, G., \& Weaver, H. A. 1984, ApJ, 285, 858

Combi, M. R., Bertaux, J. L., Quémerais, E., Ferron, S., \& Mäkinen, J. T. T. 2011, ApJ, 734, L6

Combi, M. R., Mäkinen, T. T., Bertaux, J. L., Quémerais, E., \& Ferron, S. 2019, Icarus, 317, 610

Combi, M. R., Mäkinen, T., Bertaux, J. L., et al. 2020, Planet. Sci. J., 1, 72 
Combi, M. R., Shou, Y., Mäkinen, T., et al. 2021, Icarus, 365, 114509 Cordiner, M. A., Milam, S. N., Biver, N., et al. 2020, Nat. Astron., 4, 861 Coulson, I. M., Liu, F.-C., Cordiner, M. A., et al. 2020, AJ, 160, 182 Crovisier, J. 1987, A\&AS, 68, 223

Crovisier, J., \& Le Bourlot, J. 1983, A\&A, 123, 61

Debout, V., Bockelée-Morvan, D., \& Zakharov, V. 2016, Icarus, 265, 110

Dello Russo, N., Kawakita, H., Vervack, R. J., \& Weaver, H. A. 2016, Icarus, 278,301

Dello Russo, N., Kawakita, H., Bonev, B. P., et al. 2020, Icarus, 335, 113411

Despois, D., Gerard, E., Crovisier, J., \& Kazes, I. 1981, A\&A, 99, 320

DiSanti, M. A., Bonev, B. P., Gibb, E. L., et al. 2016, ApJ, 820, 34

DiSanti, M., Saki, M., Gibb, E., et al. 2019, EPSC-DPS Joint Meeting, 2019, 822

Dubernet, M. L., \& Quintas-Sánchez, E. 2019, Mol. Astrophys., 16, 100046

Dumouchel, F., Faure, A., \& Lique, F. 2010, MNRAS, 406, 2488

Eisner, N. L., Knight, M. M., Snodgrass, C., et al. 2019, AJ, 157, 186

Faggi, S., Villanueva, G. L., Mumma, M. J., \& Paganini, L. 2018, AJ, 156, 68

Farnham, T. L., Kelley, M. S. P., Knight, M. M., \& Feaga, L. M. 2019, ApJ, 886, L24

Farnham, T. L., Knight, M. M., Schleicher, D. G., et al. 2021, Planet. Sci. J. 2,7

Faure, A., Varambhia, H. N., Stoecklin, T., \& Tennyson, J. 2007, MNRAS, 382, 840

Faure, A., Lique, F., \& Loreau, J. 2020, MNRAS, 493, 776

Fink, U., Doose, L., Rinaldi, G., et al. 2016, Icarus, 277, 78

Fitzsimmons, A., Hainaut, O., Meech, K. J., et al. 2019, ApJ, 885, L9

Fray, N., Bénilan, Y., Cottin, H., Gazeau, M. C., \& Crovisier, J. 2005, Planet. Space Sci., 53, 1243

Gersch, A. M., \& A'Hearn, M. F. 2014, ApJ, 787, 36

Giorgini, J. D., Yeomans, D. K., Chamberlin, A. B., et al. 1996, AAS/Div. Planet. Sci. Meeting Abs., \#28, 25.04

Gordon, I. E., Rothman, L. S., Hill, C., et al. 2017, J. Quant. Spectr. Rad. Transf., 203, 3

Hänni, N., Altwegg, K., Pestoni, B., et al. 2020, MNRAS, 498, 2239

Haser, L. 1957, Bulletin de la Societe Royale des Sciences de Liege, 43, 740

Hertel, I. V., \& Schulz, C.-P. 2015, Atoms, Molecules and Optical Physics 2 (Berlin, Heidelberg: Springer)

Howell, E. S., Lejoly, C., Taylor, P. A., et al. 2017, AAS/Div. Planet. Sci. Meeting Abs., \#49, 414.24

Huebner, W. F., \& Mukherjee, J. 2015, Planet. Space Sci., 106, 11

Huebner, W. F., Keady, J. J., \& Lyon, S. P. 1992, Ap\&SS, 195, 1

Ip, W. H. 1985, Adv. Space Res., 5, 233

Khan, Y., Gibb, E. L., Bonev, B. P., et al. 2021, Planet. Sci. J., 2, 20

Läuter, M., Kramer, T., Rubin, M., \& Altwegg, K. 2020, MNRAS, 498, 3995

Lecacheux, A., Biver, N., Crovisier, J., et al. 2003, A\&A, 402, L55

Lis, D. C., Bockelée-Morvan, D., Boissier, J., et al. 2008, ApJ, 675, 931
Lis, D. C., Biver, N., Bockelée-Morvan, D., et al. 2013, ApJ, 774, L3

Lis, D. C., Bockelée-Morvan, D., Güsten, R., et al. 2019, A\&A, 625, L5

Lovell, A. J., Amason, C., Howell, E. S., et al. 2017, AAS/Div. Planet. Sci. Meeting Abs., 49, 420.04

McKay, A. J., DiSanti, M. A., Cochran, A. L., et al. 2021, Planet. Sci. J., 2, 21

Meech, K. J., Schambeau, C. A., Sorli, K., et al. 2017, AJ, 153, 206

Moulane, Y., Jehin, E., Opitom, C., et al. 2018, A\&A, 619, A156

Moulane, Y., Jehin, E., José Pozuelos, F., et al. 2019, EPSC-DPS Joint Meeting, 2019, 1036

Müller, H. S. P., Thorwirth, S., Roth, D. A., \& Winnewisser, G. 2001, A\&A, 370, L49

Mumma, M. J., \& Charnley, S. B. 2011, ARA\&A, 49, 471

Mumma, M. J., DiSanti, M. A., Dello Russo, N., et al. 2003, Adv. Space Res., 31,2563

Opitom, C., Yang, B., Selman, F., \& Reyes, C. 2019, A\&A, 628, A128

Paganini, L., Villanueva, G. L., Lara, L. M., et al. 2010, ApJ, 715, 1258

Paradowski, M. L. 2020, MNRAS, 492, 4175

Pickett, H. M., Poynter, R. L., Cohen, E. A., et al. 1998, J. Quant. Spectr. Rad. Transf., 60, 883

Rabli, D., \& Flower, D. R. 2010, MNRAS, 406, 95

Roth, N. X., Gibb, E. L., Bonev, B. P., et al. 2018, AJ, 156, 251

Roth, N. X., Bonev, B. P., DiSanti, M. A., et al. 2021a, Planet. Sci. J., 2, 54

Roth, N. X., Milam, S. N., Cordiner, M. A., et al. 2021b, ApJ, 921, 14

Roth, N. X., Milam, S. N., Cordiner, M. A., et al. 2021c, Planet. Sci. J., 2, 55

Rubin, M., Hansen, K. C., Gombosi, T. I., et al. 2009, Icarus, 199, 505

Rubin, M., Altwegg, K., Balsiger, H., et al. 2019, MNRAS, 489, 594

Rybicki, G. B., \& Hummer, D. G. 1991, A\&A, 245, 171

Saki, M., Gibb, E. L., Bonev, B. P., et al. 2020, AJ, 160, 184

Saki, M., Gibb, E. L., Bonev, B. P., et al. 2021, AJ, 162, 145

Schleicher, D. G., Knight, M. M., Eisner, N. L., \& Thirouin, A. 2019, AJ, 157, 108

Schöier, F. L., van der Tak, F. F. S., van Dishoeck, E. F., \& Black, J. H. 2005, A\&A, 432, 369

Sekanina, Z. 2017, ArXiv e-prints [arXiv:1712.03197]

Springmann, A., Harris, W., Ryan, E., \& Lejoly, C. 2019, EPSC-DPS Joint Meeting, 2019, 1904

van der Tak, F. F. S., Black, J. H., Schöier, F. L., Jansen, D. J., \& van Dishoeck, E. F. 2007, A\&A, 468, 627

Venkataramani, K., \& Ganesh, S. 2018, Euro. Planet. Sci. Congress, 2018, 1220

Wang, Z., Zhang, S.-B., Tseng, W.-L., et al. 2020, AJ, 159, 240

Wirström, E. S., Geppert, W. D., Hjalmarson, A., et al. 2011, A\&A, 533, A24

Wirström, E. S., Lerner, M. S., Källström, P., et al. 2016, A\&A, 588, A72

Xie, X., \& Mumma, M. J. 1992, ApJ, 386, 720

Zakharov, V., Bockelée-Morvan, D., Biver, N., Crovisier, J., \& Lecacheux, A. 2007, A\&A, 473, 303 

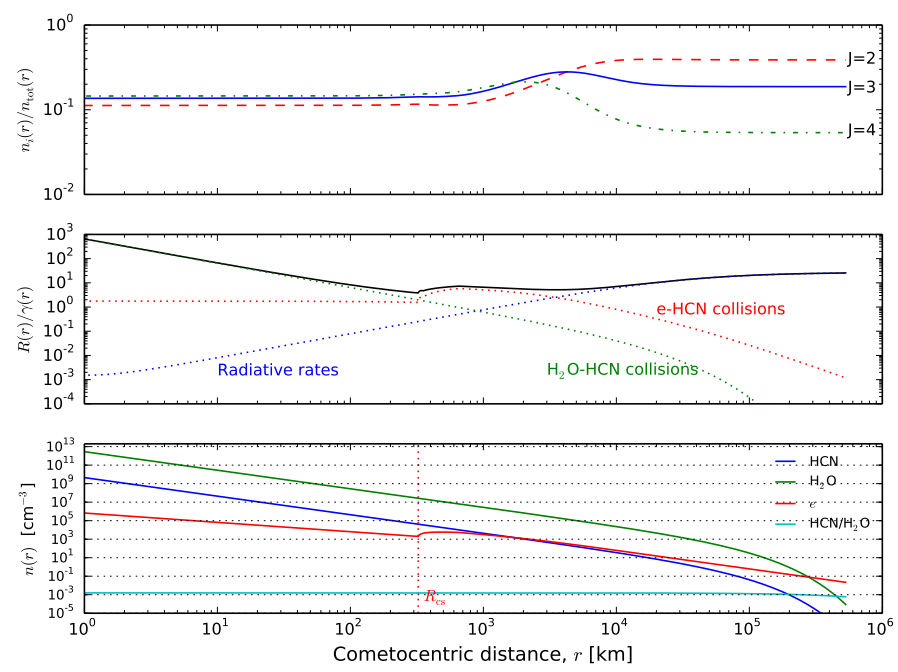

Fig. A.1. Time-dependent HCN modelling with parameters corresponding to the fragment $\mathrm{B}$ of $73 \mathrm{P} / \mathrm{Sch}$ wassmann-Wachmann. The upper plot shows the fractional level populations for levels $J=2,3,4$ as a function of cometocentric distance. The middle plot shows the relative importance of different downward rates for the $\mathrm{HCN} J=3-2$ transition As indicated, the included rates are radiative (excluding IR pumping rates), and collisional rates both for electrons and water molecules. The sum of these rates is included as a black line. They are normalized with the time-dependent rate due to destruction and constant expansion; see Eq. (5). The bottom panel depicts the radial density variations. The blue curve is the total $\mathrm{HCN}$ density and the green curve represents the water density behaviour. The electron density, $n_{\mathrm{e}}(r)$, is drawn in red. Also the $\mathrm{HCN} / \mathrm{H}_{2} \mathrm{O}$ ratio is shown. It is not entirely flat, because when the photodissociation becomes important in the outer coma, $\mathrm{HCN}$ and $\mathrm{H}_{2} \mathrm{O}$ molecules are destroyed with slightly different rates.

\section{Appendix A: Validation of the time-dependent model}

As a starting point for the validation of our time-dependent model described in Sect. 4, we adopt the case for comet 73P/Schwassmann-Wachmann, hereafter 73P, which broke into fragments during its 2006 apparition. Paganini et al. (2010) observed the HCN $J=3-2$ and $J=4-3$ lines in 73P on May 12, 2006, using the Heinrich Hertz Submillimeter Telescope (HHSMT). Contemporary Caltech Submillimeter Observatory (CSO) HCN observations of 73P/B were also made (Lis et al. 2008). Paganini et al. (2010) also performed comprehensive modelling of the $\mathrm{HCN}$ emission to which we can compare our modelling results. We adopt the values for the fragment B that these authors used: $Q_{\mathrm{HCN}}=3 \times 10^{25} \mathrm{~mol} \mathrm{~s}^{-1}, Q_{\mathrm{H}_{2} \mathrm{O}}=1.9 \times$ $10^{28} \mathrm{~mol} \mathrm{~s}^{-1}, T=90 \mathrm{~K}$, and $v_{\mathrm{e}}=0.53 \mathrm{~km} \mathrm{~s}^{-1}$. To further mimic their setup, we neglect hfs and include effective pumping rates. In the upper panel of Fig. A.1 we show the HCN fractional level populations for $J=2,3,4$ as a function of cometocentric radius.

The appearance of the level population variation looks very similar to that of Fig. 1 in Paganini et al. (2010), suggesting that any time-dependent effects are not significant in this particular case. Our late time values are about a factor 1.2-1.5 larger than those of Paganini et al. (2010). The middle panel in this figure depicts the relative importance of the different $\mathrm{HCN}$ excitation mechanisms (for $J=3-2$ ). In the inner coma, collisions with water molecules are most important, while in the outer coma, radiative processes dominate. It between, as shown by Xie \& Mumma (1992), collisions by electrons are the most important excitation mechanism.

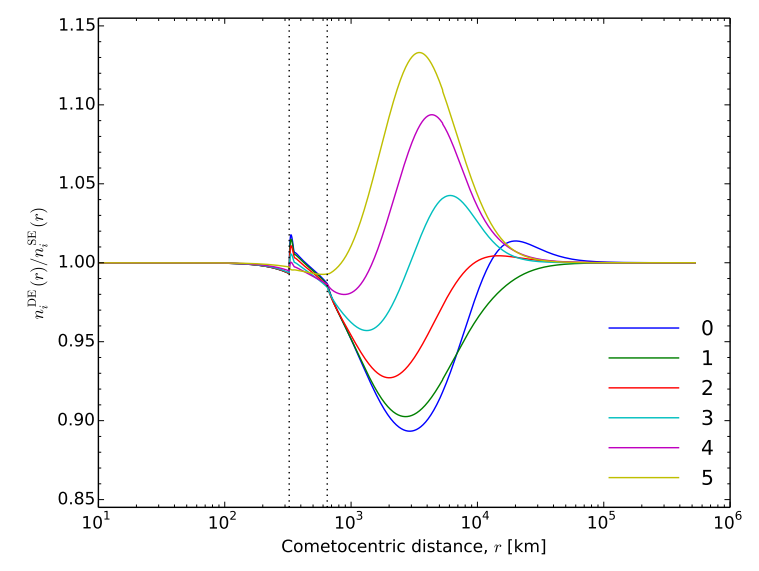

Fig. A.2. HCN level ( $J=0$ to 5 ) population variation as a function of cometocentric radius, for the time-dependent modelling (see Fig. A.1) adopting the parameters for fragment B of 73P/SchwassmannWachmann during its 2006 passage. The level populations have been normalised to their SE-values to visualise time-dependent effects. The vertical dotted lines correspond to radii $R_{\mathrm{CS}}$ and $2 R_{\mathrm{CS}}$ between which the electron temperature increases from $90 \mathrm{~K}$ to $10^{4} \mathrm{~K}$.

The different downward rates in the middle panel of Fig. A.1 have been normalised with the rate due to expansion and destruction, $\gamma$ in Eq. (5). When this ratio is around 1 or smaller, we may see time dependency effects. As visualised in the bottom panel of Fig. A.1, where the radial density variations of water, $\mathrm{HCN}$, and electrons are shown, the electron properties change very rapidly at the contact surface radius, $R_{\mathrm{CS}}=324 \mathrm{~km}$. Here the electron temperature $T_{\mathrm{e}}$ rises very steeply from the comet temperature to around $10^{4} \mathrm{~K}$ (Ip 1985; Bensch \& Bergin 2004), accompanied with an increase in electron density.

It is around a cometocentric radius near $R_{\mathrm{CS}}$ (see the middle panel in Fig. A.1) where we should start to see the timedependent effects on the excitation (cf. Chin \& Weaver 1984). To investigate this in more detail, we display in Fig. A.2 the ratio of the time dependent level populations $(J=0$ to 5$)$ to those valid in the SE-limit. The level population ratios are very close to 1 up to about $r=100 \mathrm{~km}$ (cf. Bockelée-Morvan et al. 1984) and from radii larger than about $r=(4-5) \times 10^{4} \mathrm{~km}$. After the sharp increase in $T_{\mathrm{e}}$ from $90 \mathrm{~K}$ to $10^{4} \mathrm{~K}$ over the interval $\left[R_{\mathrm{CS}}, 2 R_{\mathrm{CS}}\right.$ ], the level populations deviate from the SE-values during the settling - in this case at most up to $7 \%$ for $J=2$. The low- $J$ level populations settle a little later than the high- $J$ level populations because their $A$-coefficients are smaller. This deviation is not directly related to the change in electron properties but to that of the normalized rates (see middle panel in Fig. A.1), which are close to 1 . Due to its large electric dipole moment, HCN rotational transitions have large $A$-coefficients and we expect that for molecules with smaller spontaneous rates (e.g. CO), the settling of the level populations will take place further out in the coma. The deviations from the SE populations will be larger if $Q_{\mathrm{H}_{2} \mathrm{O}}$ is reduced because $\mathrm{HCN}-\mathrm{H}_{2} \mathrm{O}$ collisions will be less effective in settling the populations. For instance, using $Q_{\mathrm{H}_{2} \mathrm{O}}=$ $1 \times 10^{28} \mathrm{~mol} \mathrm{~s}^{-1}$ in our test case, the time-dependent $J=2$ population deviations reach $9 \%$ and for $Q_{\mathrm{H}_{2} \mathrm{O}}=4 \times 10^{28} \mathrm{~mol} \mathrm{~s}^{-1}$ they are at the 5\% level. By reducing the number of electron collisions (by lowering $x_{n_{\mathrm{e}}}$ from 0.3 to 0.15 ) the effects are less clear. Firstly, the initial time-dependent deviation at $R_{\mathrm{CS}}$ will be less pronounced but the settling at larger radii, $10^{3}-10^{4} \mathrm{~km}$, will show larger deviations. Increasing the number of electron collisions will reduce the slower time-dependent behaviour (and increase the deviation at $R_{\mathrm{CS}}$ ). 